\title{
The Composition of the Protosolar Disk and the Formation Conditions for Comets
}

\author{
K. Willacy ${ }^{1}$ C. Alexander ${ }^{2}$ M. Ali-Dib ${ }^{3}$. C. Ceccarelli, ${ }^{4,5}$ - S.B. Charnley ${ }^{6}$. \\ M. Doronin ${ }^{7,8}$ - Y. Ellinger ${ }^{7}$ - P. Gast ${ }^{9}$ - E. Gibb ${ }^{10}$ - S.N. Milam ${ }^{6}$ - O. Mousis ${ }^{11}$ \\ F. Pauzat ${ }^{7}$. C. Tornow ${ }^{9}$. E.S. Wirström ${ }^{12}$ - E. Zicler ${ }^{7}$
}

Received: 6 November 2014 / Accepted: 22 May 2015

(C) Springer Science+Business Media Dordrecht 2015

\begin{abstract}
Conditions in the protosolar nebula have left their mark in the composition of cometary volatiles, thought to be some of the most pristine material in the solar system. Cometary compositions represent the end point of processing that began in the parent molecular cloud core and continued through the collapse of that core to form the protosun and the solar nebula, and finally during the evolution of the solar nebula itself as the cometary bodies were accreting. Disentangling the effects of the various epochs on the final composition of a comet is complicated. But comets are not the only source of information about the so-
\end{abstract}

$凶$ K. Willacy

karen.willacy@jpl.nasa.gov

1 MS 169-506, NASA Jet Propulsion Laboratory, California Institute of Technology, 4800 Oak Grove Drive, Pasadena, CA 91109, USA

2 MS 321-590, NASA Jet Propulsion Laboratory, California Institute of Technology, 4800 Oak Grove Drive, Pasadena, CA 91109, USA

3 Institut UTINAM, CNRS/INSU, UMR 6213, Université de Franche-Comté, 41 bis Avenue de l'Observatoire, BP1615, 25010 Besançon, France

4 IPAG, Univ. Grenoble Alpes, 38000 Grenoble, France

5 IPAG, CNRS, 38000 Grenoble, France

6 Astrochemistry Laboratory, Mail Code 691, NASA Goddard Space Flight Center, Greenbelt, MD 20771, USA

7 Laboratoire de Chimie Théorique (LCT), Sorbonne Université, UPMC Univ Paris 06, CNRS UMR, 7616, 75252 Paris CEDEX 05, France

8 Laboratoire de Physique Moléculaire pour l'Atmosphère et l'Astrophysique (LERMA/LPMAA), Sorbonne Université, UPMC Univ Paris 06, CNRS UMR, 7092, 75252 Paris CEDEX 05, France

9 Institute of Planetary Research (DLR), Rutherfordstraße 2, 12489 Berlin, Germany

10 Department of Physics and Astronomy, University of Missouri - St. Louis, St. Louis, MO 63121, USA

11 LAM (Laboratoire d'Astrophysique de Marseille) UMR 736, Aix-Marseille Université, CNRS, 13388, Marseille, France

12 Onsala Space Observatory, Department of Earth and Space Sciences, Chalmers University of Technology, 439 92, Onsala, Sweden 
lar nebula. Protostellar disks around young stars similar to the protosun provide a way of investigating the evolution of disks similar to the solar nebula while they are in the process of evolving to form their own solar systems. In this way we can learn about the physical and chemical conditions under which comets formed, and about the types of dynamical processing that shaped the solar system we see today.

This paper summarizes some recent contributions to our understanding of both cometary volatiles and the composition, structure and evolution of protostellar disks.

Keywords Protostellar disks $\cdot$ Solar nebula $\cdot$ Comets $\cdot$ Chemistry

$\begin{array}{ll}\text { Abbreviations } & \\ \text { AGB } & \text { Asymptotic giant branch } \\ \text { ALMA } & \text { Atacama Large Millimeter Array } \\ \text { CAI } & \text { Calcium-rich aluminium-rich inclusion } \\ \text { FUN } & \text { Fractionated and unknown nuclear isotopic effects } \\ \text { ISM } & \text { Interstellar medium } \\ \text { ISRF } & \text { Interstellar radiation field } \\ \text { IRAM } & \text { Institut de Radioastronomie Millimétrique telescope } \\ \text { MRI } & \text { Magneto-rotational instability } \\ \text { MHD } & \text { Magnetohydrodynamical } \\ \text { OPR } & \text { Ortho-to-para ratio } \\ \text { PSN } & \text { Protosolar nebula } \\ \text { SLR } & \text { Short-lived radionuclide } \\ \text { Gaseous inner disk } & \text { Inside the iceline } \\ \text { Inner disk } & \text { Inside 35 AU } \\ \text { Outer disk } & \text { Outside 35 AU }\end{array}$

\section{Introduction}

Cometary volatiles are some of the most pristine of solar system materials, having remained relatively unprocessed since the comets formed in the inner solar system some 4.2 billion years ago. Consequently they retain in their composition signatures of the chemical and physical conditions under which they formed. Observations of cometary comae provide the mixing ratios or relative abundances of various species and have demonstrated that these can vary greatly between comets, e.g., A'Hearn et al. (2012) and Mumma and Charnley (2011) and references therein, with little correlation with dynamical family. The origin of these compositional variations is not well understood but must lie in the physical and chemical processes that were active at the time and location at which the cometary volatiles were formed. To understand these processes requires not only a study of the comets themselves but also of protostellar disks similar to the one from which our solar system formed. Observations of protostellar disks allow for the important disk processes to be investigated while they are occurring and can test models of the evolution of the early solar system.

Some key questions that can potentially be answered by interdisciplinary investigations involving both protostellar disk modeling and observations, and the observations of comets are:

1. Can synergy between protoplanetary disk modeling and compositional studies of comets inform us about the chemical history and processes in the early solar disk?

2. What is the origin of the diversity of observed cometary compositions? 
3. Where did comets form in the solar nebula?

4. Are $\mathrm{D} / \mathrm{H}$ ratios in volatile molecules and spin temperatures cosmogonic indicators for comets? If so, what can they tell us about the formation conditions in the early solar system?

5. What is the range of $\mathrm{D} / \mathrm{H}$ ratios in cometary water and can we infer the degree to which comets contributed volatiles to the early terrestrial planets?

6. Can cometary volatile abundances be used to constrain the extent of radial and vertical mixing in the early protoplanetary disk?

This paper summarizes some recent developments in our understanding of cometary compositions and their relationship to the conditions under which the comets formed. The paper is arranged as follows. Section 2 covers some observational and modeling studies of protostellar disks. Section 3 discusses fractionation processes in protostellar disks including using deuterium fractionation as a tracer of the links between comets, interstellar chemistry and nebular chemistry. Section 4 considers the origin of the nitrogen deficiency in comets and Sect. 5 discusses spin temperature as a cosmogenic indicator in comets. Section 6 presents a possible explanation for the observations of neutral sodium cometary tails. Finally, Sect. 7 summarizes this paper and briefly discusses the potential impact of Rosetta on our understanding of the formation conditions of comets.

\section{Overview of the Structure and Composition of Protostellar Disks}

Protostellar disks form during the star formation process. A molecular cloud core collapses under gravity forming a protostar in the center. As material continues to fall inwards conservation of angular momentum results in the formation of a disk around the central protostar. In the early stages, the disks are gas-dominated with masses $\sim 1 \%$ of the mass of the protostar. They rotate about the protostar with a Keplerian velocity profile. Disks contain a wide range of physical conditions, ranging from cold ( $\sim 10-20 \mathrm{~K}$ in the outer disk midplane) to hot (with gas temperatures up to several thousand $\mathrm{K}$ in the surface layers). Irradiation conditions also vary, with the surface layers experiencing the full force of the stellar UV field, but with little radiation reaching the midplane. Consequently there are a wide variety of chemical conditions depending on location within the disk. The star and disk formation process, and the chemistry and dynamics of disks are discussed in more detail in recent reviews by Dutrey et al. (2014); Pontoppidan et al. (2014) and Turner et al. (2014).

Disks can be very turbulent places. Turbulence is difficult to detect directly but evidence for its effects can be seen in the presence of small dust grains high above the disk midplane. Without turbulence these grains would quickly be removed from these high altitudes by settling (Dullemond and Dominik 2004). The dynamical motions also drive collisions between grains, which can lead to both grain growth, eventually forming planetesimals, and to creation of new small grains or dust. With grain growth, the grains will eventually reach a size at which their motions decouple from those of the gas and they begin to settle under the influence of gravity to the midplane, where they can undergo further collisions and growth to form planetesimals. The removal of grains from the surface layers allows the stellar UV to penetrate deeper into the disk, changing its composition. Turbulence can also drive chemical changes by bringing together material processed in different parts of the disk and conversely chemistry can affect the dynamics by controlling the ionization levels. Consequently dynamics and chemistry strongly influence each other (see Sect. 2.3.2). 

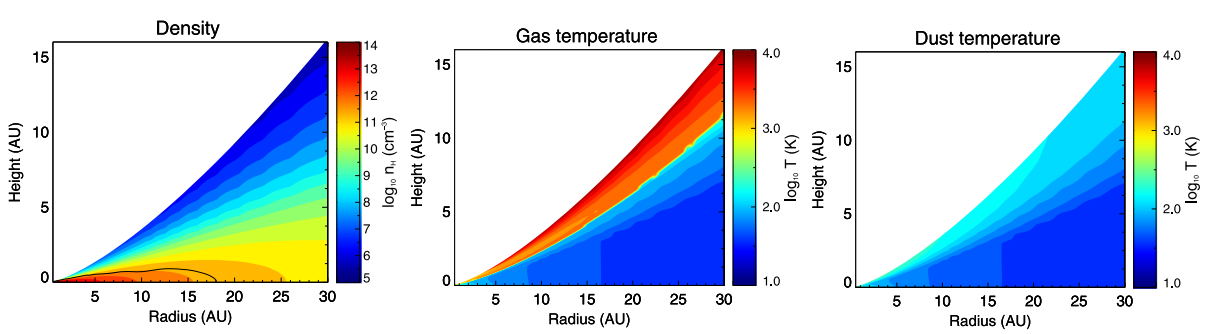

Fig. 1 The physical structure of a typical T Tauri disk. Shown are the density (left), gas temperature (center) and dust temperature (right). The density and dust temperatures are calculated by a hydrodynamical model of Paola D'Alessio with a similar mass and surface density profile to the minimum mass solar nebula. The gas temperature is determined via thermal balance and is coupled to the dust temperature throughout most of the disk, but is much hotter in the surface layers due to the photoelectric effect. The black contour line on the density plot indicates the likely location of the dead zone, determined by the location where the ionization level $x(e)<10^{-12}$. In this region the turbulent motions driven by MRI will be severely inhibited

In recent years protostellar disks have been an area of active research, with their molecular content, dust properties, and physical conditions being studied in detail. The observational work has been coupled with simulations that can interpret the observations and make predictions about those regions of the disks which are not amenable to direct observational study. This synergy of the observations and modeling work has revealed a wealth of information about the chemistry and physics of disks, and in turn has informed our understanding of the early history of our own solar system. This section reviews some of the recent observational and modeling work related to protostellar disks, concentrating on the early stages of evolution when the disk is still gas-dominated (the T Tauri phase).

\subsection{The Structure of a Protostellar Disk}

Protostellar disks are characterized by strong radial and vertical gradients in temperature and density (Fig. 1). The stellar UV field controls the temperature and hence the chemistry throughout much of the disk. The penetration of the UV into the disk is governed by the opacity which is mainly provided by dust absorption. In the surface layers, where the UV field is unattenuated, the photoelectric effect can efficiently heat the gas to much higher temperatures than the dust. Close to the midplane, where the UV field is reduced, the dust and grain temperatures are coupled.

These gradients in physical conditions lead to a wide variety of chemical environments (Fig. 2). Models have found that outside of the water ice line the disk can be divided vertically into three chemically distinct layers. At the surface, the disk is dominated by the effects of UV. Ices are desorbed (either thermally or by photodesorption) and efficient photodissociation means that the gas is mainly composed of atoms and their ions. In the midplane most molecules are frozen out onto the surfaces of dust grains. Between these two layers is a region where the grains are warm enough to allow at least some molecules to thermally desorb and which is shielded enough that photodissociation is inefficient, allowing the molecules to survive once in the gas. It is this warm molecular layer that is detected in most gas phase observations of the outer regions of protostellar disks (see Sect. 2.2).

As the distance to the protostar decreases the disk warms up. In the midplane, the ice mantles will begin to desorb, with different molecules being released at different radii, depending on their binding energies - the most volatile species being released at larger radii than the less volatile ones. This results in a series of ice lines, which mark the transition 


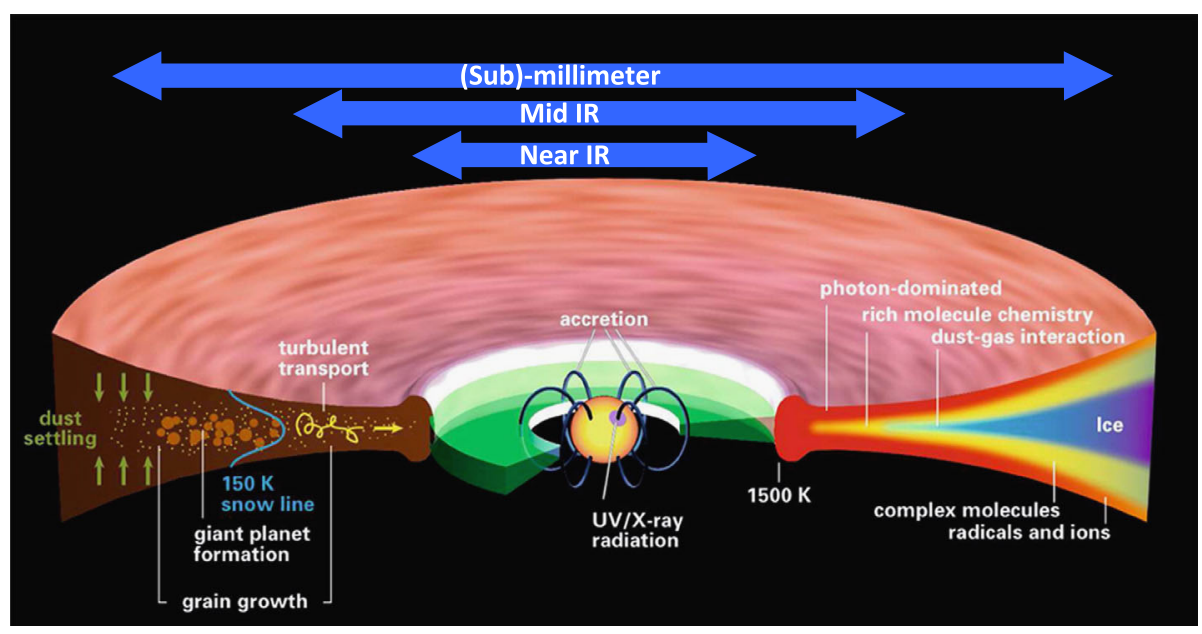

Fig. 2 The physical and chemical environment of a 1-5 Myr old protostellar disk around a Sun-like star. Reprinted from Henning and Semenov (2013) with permission. (C)(2013) American Chemical Society

from ice to vapor for each molecule (see Sect. 2.4). The icelines are curved, and in the outer disk are almost horizontal with the molecule existing as ice below this line and as gas above it (see Pontoppidan et al. 2014). Once inside the water iceline (within a few AU of the protostar) the molecules are all in the gas phase.

Figure 2 also illustrates some of the main physical processes that are active in disks. In particular, turbulence is a crucial process, enabling dust coagulation and driving chemical changes by mixing material processed under different physical conditions within the disk. What drives this turbulence is still a matter of debate but one of the main candidate processes is the magneto-rotational instability (MRI; e.g., Balbus and Hawley 1991; Hawley and Balbus 1991; Hawley et al. 1996) which can efficiently transport angular momentum in ionized accretion disks. A minimum level of ionization is required (in the minimum mass solar nebula the ionization fraction, $x(e)$, must be $>10^{-12}$ at $1 \mathrm{AU}$; Inutsuka and Sano 2005). This can be difficult to achieve in the shielded midplane regions of a protostellar disk, where the high column densities prevent UV and cosmic rays from penetrating. Hence there are regions in the disk where the MRI is not active. These are known as "dead zones" and they are predicted to occur throughout much of the midplane in the planet-forming region. The approximate location of the dead zone in the disk shown in Fig. 1 is indicated by the black contour line in the density plot.

The dependence of MRI-driven turbulence on the ionization means that there is a close link between the chemistry and the dynamics. The chemistry determines the ionization level and therefore the strength of the turbulence, but the dynamical motions themselves can drive chemical changes through mixing. This in turn affects the ionization and consequently the dynamics (e.g., Inutsuka and Sano 2005; Ilgner and Nelson 2006, 2008; Turner et al. 2007). Hence the evolution of chemistry and dynamics are inextricable linked.

\subsection{Observations of Protostellar Disks}

Protostellar disks have been observed at wavelengths from the UV to the millimeter. Each wavelength can probe a different region of the disk. The majority of information about the molecular composition of disks comes from the infrared and the (sub)-millimeter. The disk is 
mostly optically thick in the infrared, therefore these observations are restricted to the warm gaseous region inside of the iceline or to the surface layers. At these wavelengths gaseous molecules can be observed in both absorption and emission. Ices can also be observed in absorption if illuminated by the central star in an edge-on disk, e.g., Pontoppidan et al. (2005), but the spectra can be difficult to interpret due to contributions from material outside of the disk. Near- and mid-infrared observations are most sensitive to the inner regions of disks, while the far-infrared can probe the surface layers at larger radii (see Henning and Semenov 2013).

Spitzer, operating in the mid-infrared, has shown that inside of the iceline the disk is chemically rich, with the terrestrial planet-forming region containing organics such as $\mathrm{C}_{2} \mathrm{H}_{2}$, $\mathrm{HCN}$, as well as $\mathrm{CO}, \mathrm{CO}_{2}$, water and $\mathrm{OH}$ (e.g., Carr and Najita 2008; Salyk et al. 2008; Pontoppidan et al. 2010). The detected molecules are the result of a rich gas phase molecular chemistry, in a region where temperatures range from a few hundred kelvin up to $1000 \mathrm{~K}$, and densities are high $\left(>10^{8} \mathrm{~cm}^{-3}\right)$.

Spitzer observations of the gaseous inner disk have also been used to make inferences about the conditions beyond the iceline. For example, Najita et al. (2013) found the $\mathrm{HCN} / \mathrm{H}_{2} \mathrm{O}$ ratio inside of the iceline to be correlated with disk mass, suggesting that this ratio may reflect changes to the $\mathrm{C} / \mathrm{O}$ ratio induced by the formation of icy planetesimals outside of the iceline. Outside of the iceline much of the oxygen is tied up in water ice, whereas the carbon is mainly in the gas phase in the form of more volatile molecules such as CO. Planetesimals outside of the iceline will form from grains coated with water ice. Since the planetesimals do not move about the disk as easily the small grains and the gas, this will trap the oxygen outside of the iceline and the material that moves inwards to the inside of the iceline will be enriched in carbon. Consequently the gaseous inner disk will be enriched in organics compared to water. Since more massive disks are likely to form planetesimals more efficiently than their lower mass counterparts they will have a higher $\mathrm{C} / \mathrm{O}$ ratio inside of the iceline.

In the far-infrared, Herschel has detected a number of molecules including cold water in the outer disks of TW Hya (Hogerheijde et al. 2011) and DG Tau (Podio et al. 2013). In both cases the water emission comes from a region of the disk where grain temperatures are low enough for this molecule to be expected to be frozen onto dust grains. Its presence in the gas can be explained by photodesorption driven by the stellar UV field (Willacy and Langer 2000; Dominik et al. 2005). Hogerheijde et al. (2011) found that the predicted flux from photodesorbed water to be higher than observed, and suggested that the answer to this discrepancy could be grain coagulation. As the grains grow they decouple from the gas motions and sink towards the midplane, taking their ices with them. This removes water ice from the molecular layer, leaving less to be photodesorbed, and thus reducing the gas phase abundance.

Emission lines from some molecules in the disk can be optically thin in the (sub)millimeter and so in theory these wavelengths can probe the whole of the disk. In practice, however, resolution constraints restrict these observations to $\gtrsim 30-100$ AU from the protostar, although ALMA allows observations to within a few AU of the closest protostars e.g. ALMA Partnership et al. (2015). Observations by both interferometers and single dish facilities have detected the emission from molecules in the warm molecular region and their distributions can be mapped using interferometers. Relatively simple molecules have been observed including $\mathrm{CO}$ (and its isotopologues), $\mathrm{H}_{2} \mathrm{CO}, \mathrm{CS}, \mathrm{C}_{2} \mathrm{H}, \mathrm{HCN}, \mathrm{CN}, \mathrm{HNC}, \mathrm{DCN}$, $\mathrm{HC}_{3} \mathrm{~N}, \mathrm{c}-\mathrm{C}_{3} \mathrm{H}_{2}, \mathrm{~N}_{2} \mathrm{H}^{+}, \mathrm{HCO}^{+}, \mathrm{DCO}^{+}, \mathrm{H}_{2} \mathrm{D}^{+}$(for a recent review see Dutrey et al. 2014). The fractional abundances relative to $\mathrm{H}_{2}$ are generally lower than observed in molecular clouds because of the combined effect of depletion in the cold dense midplane and photodissociation in the surface layers. Both processes reduce the gas phase molecular abundances. 
Table 1 Molecules observed in protostellar disks and the processes they trace

\begin{tabular}{ll}
\hline Process & Molecules \\
\hline Temperature & $\mathrm{CO},{ }^{13} \mathrm{CO}, \mathrm{C}^{17} \mathrm{O}, \mathrm{C}^{18} \mathrm{O}, \mathrm{H}_{2} \mathrm{CO}, \mathrm{H}_{2} \mathrm{O}, \mathrm{OH}, \mathrm{H}_{2}$ \\
Density & $\mathrm{CO}, \mathrm{H}_{2} \mathrm{CO}, \mathrm{HCO}^{+}, \mathrm{HC}_{3} \mathrm{~N}$ \\
Ionization & $\mathrm{HCO}^{+}, \mathrm{N}_{2} \mathrm{H}^{+}, \mathrm{CH}^{+}$ \\
Velocity & $\mathrm{CO}, \mathrm{CS}, \mathrm{HC}_{3} \mathrm{~N}$ \\
Photodissociation & $\mathrm{CN}, \mathrm{HCN}, \mathrm{HNC}, \mathrm{OH}, \mathrm{H}_{2} \mathrm{O}, \mathrm{C}_{2} \mathrm{H}$ \\
Photodesorption & $\mathrm{H}_{2} \mathrm{O}, \mathrm{OH}$ \\
Grain surface chemistry & $\mathrm{H}_{2} \mathrm{CO}$, complex organics, $\mathrm{CO}_{2}, \mathrm{C}_{2} \mathrm{H}_{2}$ \\
High temperature chemistry & $\mathrm{Complex} \mathrm{organics}$ \\
Deuteration & $\mathrm{HD}, \mathrm{DCO}+, \mathrm{DCN}, \mathrm{H}_{2} \mathrm{D}^{+}$ \\
\hline
\end{tabular}

In addition to the composition of the disk, the detection of molecules can also provide information about the physical conditions under which they exist and the chemical processes that are active (Table 1). Observations of molecular deuteration are especially important since they trace the thermal history of the disk (see also Sect. 3.1). Deuterated molecules, with their lower column densities, also provide a tracer of the gas in the midplane, e.g., ALMA observations of $\mathrm{DCO}^{+}$have been used to trace the location of the $\mathrm{CO}$ iceline in a Herbig AeBe disk (Mathews et al. 2013) and $\mathrm{H}_{2} \mathrm{D}^{+}$is a potential tracer of the ionization level in the midplane (Ceccarelli et al. 2004).

\subsection{Protostellar Disk Modeling}

Chemical models of protostellar disks vary in their complexity, but they all predict the threelayer structure beyond the iceline as shown in Fig. 2. The chemical simulations require as their basis, a background disk model to provide the density, temperature and UV field as a function of location in the disk. These can be provided by hydrodynamical models of a $1+1 \mathrm{D}$ steady-state $\alpha$-disk model in vertical hydrostatic equilibrium such as those of D'Alessio et al. (2005); ${ }^{1}$ (see also Fig. 1). These physical disk models are computationally intensive and are generally run independently of the chemical simulations. Current chemical models are based on detailed chemical kinetics and can contain hundreds of species (in both the gas and the ices) and thousands of reactions. These models include the ionizing effects of UV photons, cosmic rays, X-rays and short-lived radionuclides such as ${ }^{26} \mathrm{Al}$. Some models have also focused on isotopic chemistry such as deuterium (e.g., Aikawa and Herbst 1999; Woods and Willacy 2007; Willacy and Woods 2009; Kavelaars et al. 2011; Furuya et al. 2013; Albertsson et al. 2014) or carbon and oxygen isotopes (Woods and Willacy 2009, see also Sect. 3.2). The simplest models assume that the conditions in the disk did not change with time and ignore dynamical motions, although a few also consider advection e.g., Willacy et al. (1998); Aikawa et al. (1999); Woods and Willacy (2007); Woods and Willacy (2009); Willacy and Woods (2009). More recent models have included processes such as grain growth and settling, e.g., Aikawa and Nomura (2006); Fogel et al. (2011); Vasyunin et al. (2011); Akimkin et al. (2013), dynamical motions, (e.g. Ilgner et al. 2004; Willacy et al. 2006; Semenov et al. 2006; Semenov and Wiebe 2011; Ilgner and Nelson 2006; Turner et al. 2006; Aikawa 2007; Hersant et al. 2009; Heinzeller et al. 2011) and

\footnotetext{
${ }^{1}$ http://www.cfa.harvard.edu/youngstars/dalessio/.
} 


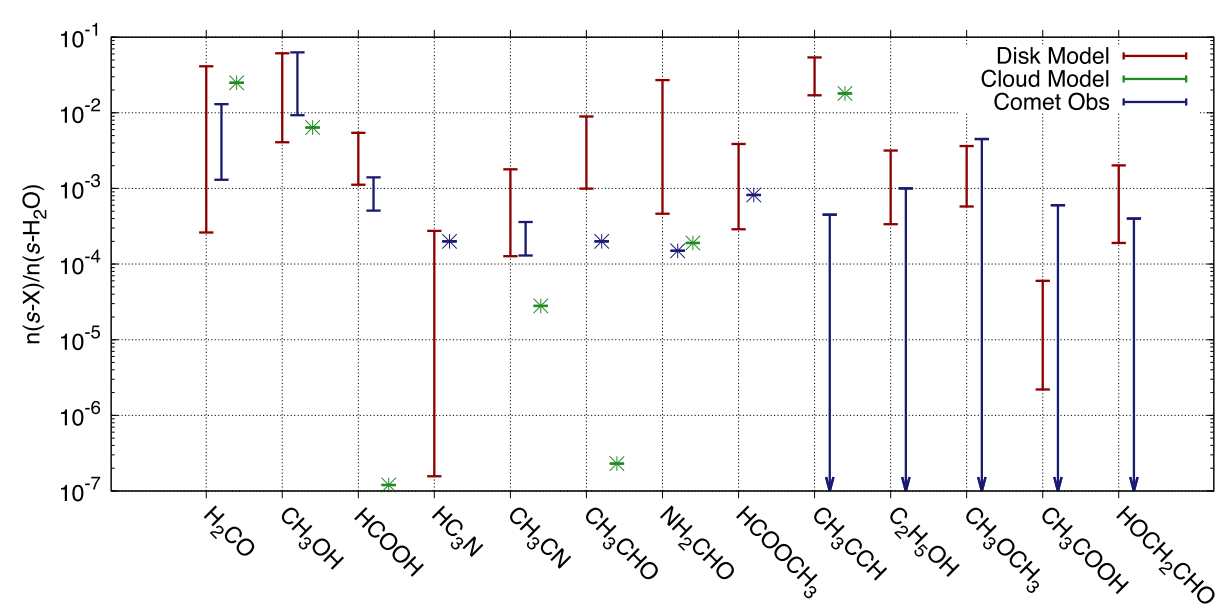

Fig. 3 Complex organics can be formed by chemistry in the ice mantles of dust grains in a protostellar disk. This figure shows the predicted range of abundances of some of these molecules relative to water ice (red lines) compared to those derived from cometary comae (blue lines; Bockelée-Morvan et al. 2004; Crovisier 2006; Crovisier et al. 2004). The green asterisks are the results from the initial molecular cloud model used as the input for the disk model. From Walsh et al. (2014)

hydrodynamical evolution of the disk structure itself (e.g. Dodson-Robinson et al. 2009; Yu et al. 2014). Here we highlight a few recent results.

\subsubsection{Formation of Complex Organics in Protostellar Disks}

Of particular interest to the chemistry of comets is the work of Walsh et al. (2014) who modeled the possible formation of complex organics through ice chemistry in protostellar disks. Figure 3 shows the range of abundances they find in ices located outside of $20 \mathrm{AU}$ in a solar-type disk. The large molecules form by sequential addition of atoms to existing mantle molecules, e.g., ethylene glycol can form by adding hydrogen, oxygen and carbon atoms to $\mathrm{CO}$ :

$$
\begin{aligned}
\mathrm{CO} & \stackrel{\mathrm{H}}{\rightarrow} \mathrm{HCO} \stackrel{\mathrm{C}}{\rightarrow} \mathrm{HC}_{2} \mathrm{O} \stackrel{\mathrm{O}}{\rightarrow} \text { OCCHO } \\
\mathrm{OCCHO} & \stackrel{\mathrm{H}}{\rightarrow} \mathrm{CHOCHO} \stackrel{2 \mathrm{H}}{\rightarrow} \mathrm{HOCH}_{2} \mathrm{CHO} \stackrel{2 \mathrm{H}}{\rightarrow}\left(\mathrm{HOCH}_{2}\right)_{2}
\end{aligned}
$$

(Charnley and Rodgers 2008, see Fig. 6). The models of Walsh et al. suggest that chemistry in disks will greatly increase the abundance of these large molecules relative to what can be created in the parent cloud. The predicted abundances relative to $\mathrm{H}_{2} \mathrm{O}$ are consistent in many cases with observations of comets (Bockelée-Morvan et al. 2004; Crovisier 2006; Crovisier et al. 2004). If these molecules can be desorbed they will also be present in the disk gas, and should be detectable by ALMA providing a test of the model.

\subsubsection{The Effects of Dynamics on the Chemistry}

There are strong links between the disk chemistry and its dynamics. The temperature, density and radiation field in a disk control the chemistry, but the chemistry (and grain evolution) in turn controls the dynamics of the disk through the ionization structure. Models have found 


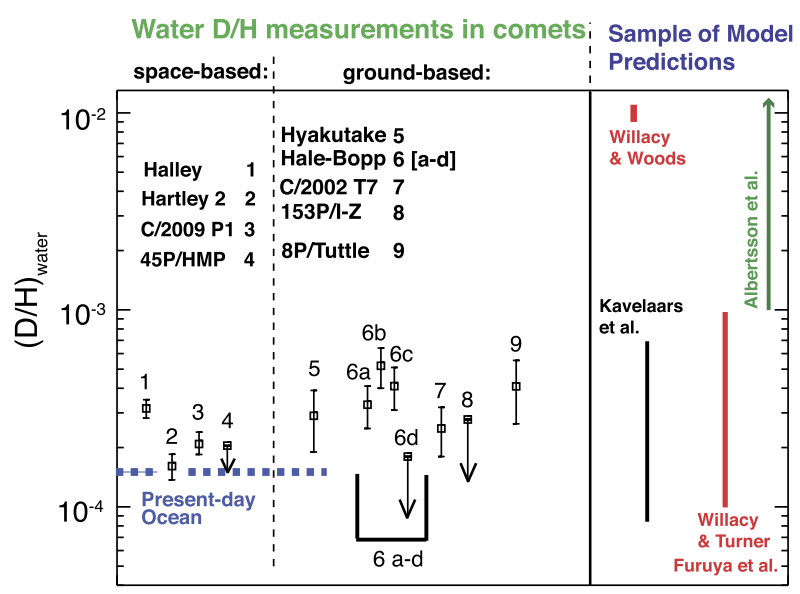

Fig. 4 Current disk models can only account for the observed D/H ratios in comets if they include mixing. The non-mixing models of Willacy and Woods (2009) and T. Millar (private communication) predict D/H ratios that are consistently higher than the observations. Albertsson et al. (2011) treat the evolution of the molecular cloud to the hot core stage only and predict $(\mathrm{D} / \mathrm{H})_{\text {water }}>10^{-3}$. Turbulent mixing is included in the disk models of Kavelaars et al. (2011) (radial mixing) and Willacy and Turner (2015) (vertical mixing at $5 \mathrm{AU}$ ) and these produce $\mathrm{D} / \mathrm{H}_{\text {water }}$ in agreement with the observations. Furuya et al. (2013) predict similar D/H ratios to Willacy and Turner (2015). References: (1) Eberhardt et al. (1995), (2) Hartogh et al. (2011), (3) Bockelée-Morvan et al. (2012), (4) Lis et al. (2013), (5) Bockelée-Morvan et al. (1998) (6a-d) Meier et al. (1998) and Crovisier et al. (2004), (7) Hutsemékers et al. (2008), (8) Biver et al. (2006), (9) Villanueva et al. (2009). Adapted from Bonev et al. (2014)

that better agreement with observations of the molecular layer in the outer disk can be obtained if vertical mixing is included, e.g., Willacy et al. (2006); Semenov et al. (2006), and more sophisticated models have now considered 2-D mixing (e.g., Heinzeller et al. 2011; Semenov and Wiebe 2011; Albertsson et al. 2014). Mixing smooths out chemical abundance gradients but also brings together species that might not otherwise exist in the same location, e.g., in the inner disk, vertical mixing combines $\mathrm{OH}$ and $\mathrm{H}_{2}$ in a region where the temperature is high enough for the neutral-neutral reaction, $\mathrm{OH}+\mathrm{H}_{2}=\mathrm{H}_{2} \mathrm{O}+\mathrm{H}$ to occur (Heinzeller et al. 2011). As a result the abundance of $\mathrm{H}_{2} \mathrm{O}$ increases.

In addition to affecting molecular abundances, mixing also changes isotopic ratios. One problem with the non-mixing models is the high deuteration levels predicted in the comet formation zone, e.g., Willacy and Woods (2009). These reflect the high levels set in the parent molecular cloud, whereas those detected in comets tend to be somewhat lower. As $\mathrm{D} / \mathrm{H}$ ratios track the thermal history of the material this suggests that at least some of the cometary ices formed at warmer temperatures than are found in the interstellar medium (ISM), e.g., Meier and Owen (1999). Figure 4 compares the observed molecular deuteration in cometary water with the predictions of several models. The non-mixing models uniformly over-predict $\mathrm{HDO} / \mathrm{H}_{2} \mathrm{O}$, but the addition of mixing (either radial or vertical) brings the ratio into closer agreement with the observations (Bonev et al. 2014).

With vertical mixing this reduction in the deuteration of water is achieved by the destruction and reformation of ice mantles. When dust grains are mixed vertically they can reach the surface layers where high temperatures and/or UV fields desorb their ices. The water molecules are dissociated and the resulting oxygen, hydrogen and deuterium atoms travel back down towards the midplane into more shielded regions where they reform molecules. The molecular deuteration depends on the formation temperature. Since the disk is warmer 


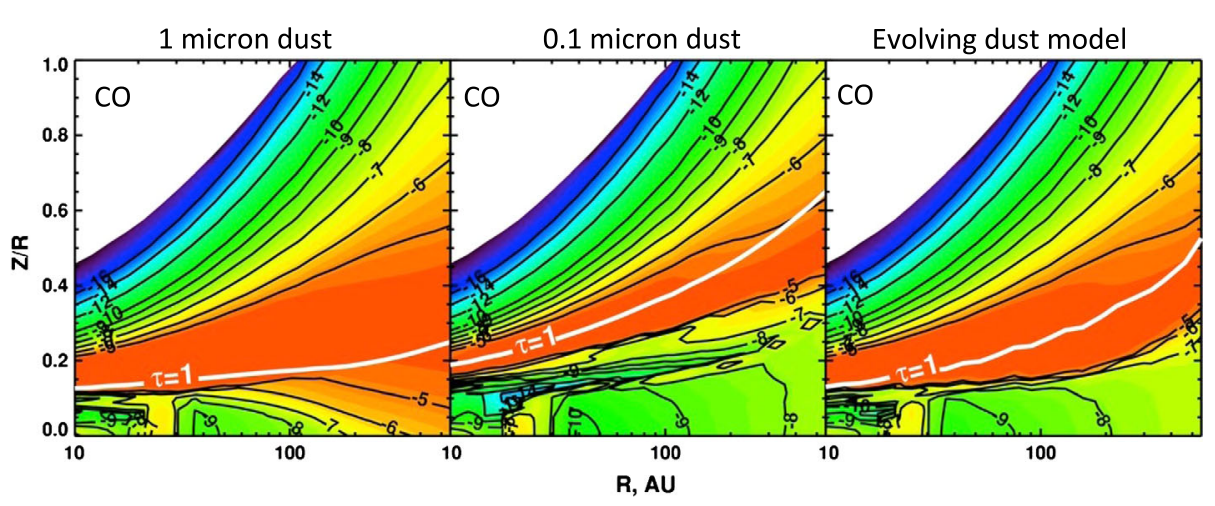

Fig. 5 The $\mathrm{CO}$ abundance distribution in a protostellar disk, showing the effects of grain growth. The highest abundances are shown in red. Grain growth reduces the opacity in the surface layers, allowing UV to penetrate further and pushing the molecular layer closer to the midplane. With 1 micron dust (left) the molecular layer is much thicker, and reaches closer to the midplane than in the model with 0.1 micron dust (center). On the right are the results from an evolving dust model where the grains are allowed to both grow and sediment. In this case the molecular layer is thicker than in the small grain model, and it moves closer to the midplane. From Vasyunin et al. (2011). CAAS. Reproduced with permission

than the $10 \mathrm{~K}$ parent molecular cloud core, the reformed water ice has a lower $\mathrm{D} / \mathrm{H}$ ratio. Hence vertical mixing can reduce the initial interstellar $\mathrm{D} / \mathrm{H}$ ratio in water ice $\sim 10^{-2}$ ) to the level detected in comets $\left(\sim 10^{-4}\right)$ (Fig. 4 and see also Furuya et al. 2013). Willacy and Turner (2015) and Furuya et al. (2013) also predict radial variations in the deuteration of water. A radial gradient in $\mathrm{HDO} / \mathrm{H}_{2} \mathrm{O}$ is also found in the (radial) mixing models of Kavelaars et al. (2011).

\subsubsection{Grain Evolution}

Grain-grain collisions in protostellar disks lead to efficient grain growth and ultimately result in the formation of planetesimals. As the grains grow they will decouple from the gas motions and sink towards the midplane. This removes grains from the upper layers of the disk, reducing the opacity and allowing UV to penetrate deeper, which increases photodissociation and heating of the newly irradiated layers, and moves the molecular layer closer to the midplane. An additional effect of grain growth is a reduction in the freezeout rate, which depends on the surface area of the grains. Figure 5 shows the effect on the chemistry of CO in the models of Vasyunin et al. (2011) (see also Aikawa and Nomura 2006; Walsh et al. 2014). Vasyunin et al. found that $\mathrm{CO}, \mathrm{CN}$ and SO, as well as the ratio of $\mathrm{C}_{2} \mathrm{H}_{2} / \mathrm{HCN}$, should be good tracers of the grain growth.

\subsection{Gas-Dust Chemistry and Icelines in Protoplanetary Disks}

Icelines in the early nebula are defined as radii at which specific molecules undergo a shift in abundance from the gas phase to the condensed phase. There are three of specific interest related to $\mathrm{H}_{2} \mathrm{O}, \mathrm{CO}_{2}$ and $\mathrm{CO}$ and these occur at radii where the temperature is approximately $150 \mathrm{~K}, 47 \mathrm{~K}$ and $20 \mathrm{~K}$ respectively (Öberg et al. 2011). Recent observations by ALMA have allowed icelines to be identified in protoplanetary disks. For example, in HD 163926 (Qi et al. 2011; de Gregorio-Monsalvo et al. 2013; 
Fig. 6 Grain surface reaction scheme forming organics via atom addition reactions to $\mathrm{CO}$ on cold dust. Molecules in red are detected both in comets and in interstellar/protostellar sources, whereas those in blue are found only in the latter. Species in black are not yet detected in space. The addition of deuterium atoms rather than hydrogen atoms to grain species can result in high levels of molecular deuteration. Adapted from Charnley and Rodgers (2008)

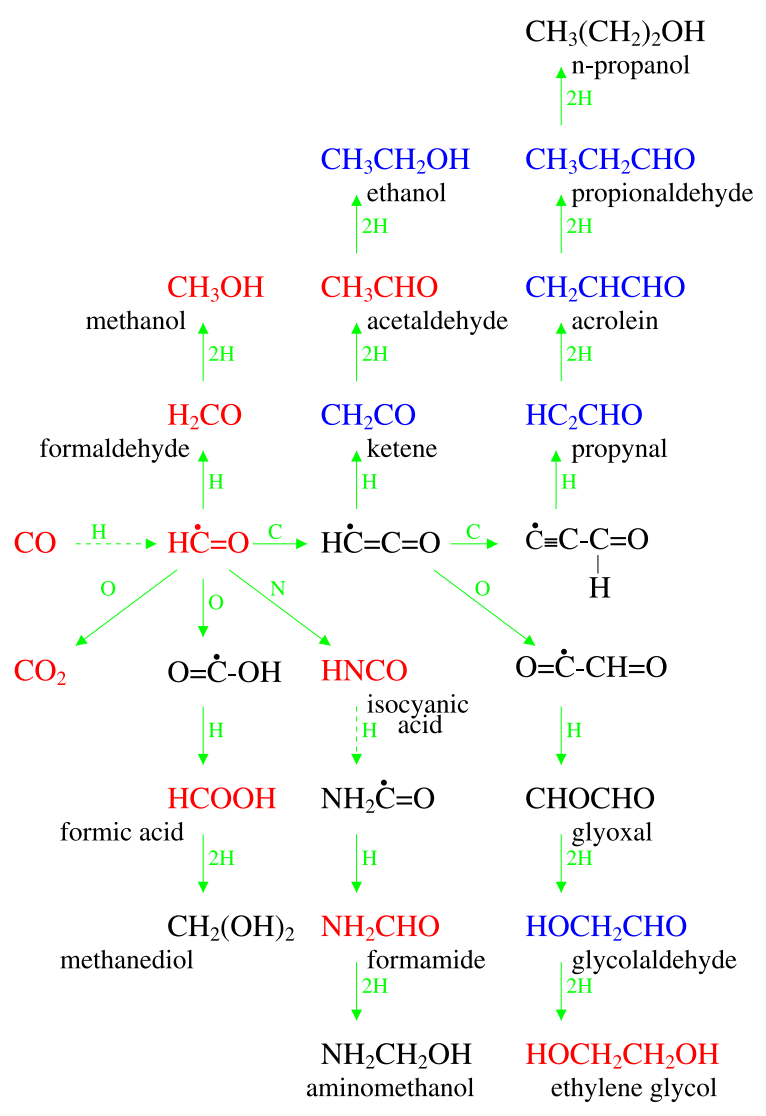

Mathews et al. 2013). The icelines associated with $\mathrm{H}_{2} \mathrm{O}, \mathrm{CO}_{2}$, and $\mathrm{CO}$ are regions of dramatic chemical change and these could be reflected in the molecular composition of the gas and ice that is transported and mixed, both vertically and radially, in the evolving disk.

The chemical signature of such an environment may be imprinted in cometary and meteoritic composition if the Sun formed in a stellar cluster containing at least one massive star (Adams 2010; Mumma and Charnley 2011). Nearby OB stars strongly affect the positions of the $\mathrm{CO}_{2}$ and $\mathrm{CO}$ icelines and the chemical structure of the disk (Walsh et al. 2013). The CO iceline occurs at tens of $\mathrm{AU}$ from the central protostar and is most accessible to observations at millimeter and submillimeter wavelengths. The physical conditions here most closely resemble those in the densest molecular cloud cores and so the related gas-dust chemistry could also be similar (Henning and Semenov 2013). This chemistry can lead to formation of many complex organic molecules on cold dust grains, as well as potentially large isotopic fractionation in both gas phase and grain-surface reactions.

For example, outside the $\mathrm{CO}$ iceline, $\mathrm{CO}$-rich ices on dust grains can undergo (tunneling) addition reactions with $\mathrm{H}$ (and D) atoms accreted from the gas (Tielens 1983; Charnley 1997). Atoms of O, C and $\mathrm{N}$ will also accrete and these processes can lead to rich organic chemistry (Fig. 6; Charnley 1997; Charnley and Rodgers 2008; Herbst and van Dishoeck 2009). Many of the proposed hydrogenation sequences have now been demonstrated in surface chemistry experiments (Watanabe and Kouchi 2008; Theulé et al. 2013) and such an ice chemistry explains the presence of the distinctive and inter-related suite of 


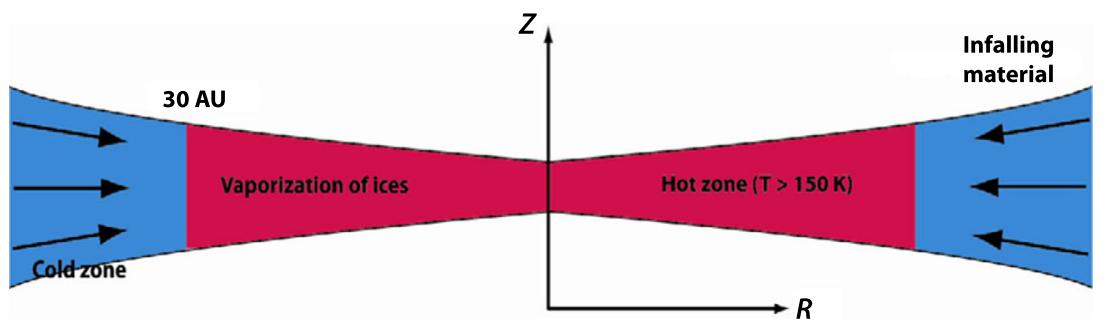

Fig. 7 Two reservoirs of ices in the PSN. A first reservoir (cold zone), located at distances higher than $\sim 30 \mathrm{AU}$, is constituted from amorphous ices coming from ISM. The second reservoir (hot zone), located within the $\sim 30 \mathrm{AU}$, is made from volatiles initially in the form of amorphous ices that were transported from ISM towards the inner and hot part of the disk. When reaching regions with temperatures higher than $\sim 150 \mathrm{~K}$, these ices vaporized. During the cooling of the disk, volatiles located in the inner 30 AU condensed again but in crystalline forms, including both pure crystalline ices and clathrates (see text)

organic molecules detected around massive and low-mass protostars (in hot cores and hot corinos, respectively), where the ice mantles have been evaporated into the hot gas (Herbst and van Dishoeck 2009). Figure 6 indicates that many of the relevant organic molecules could also be present in comets and that, if correct, ketene, ethanol, propynal, acrolein, propionaldehyde and propanol should be searched for in suitably bright apparitions. Several of the organic compounds formed in the scheme of Fig. 6 may be detectable in protoplanetary disks with ALMA. Vertical transport of ice-covered dust to the less well-shielded layers of the disk, as well as (inward) radial transport, can lead to these organic compounds being released by evaporation and/or photodesorption. This is probably the origin of the $\mathrm{H}_{2} \mathrm{CO}$ detected in HD 163296, which lies outside of the CO iceline at about 160 AU (Qi et al. 2013b).

\subsection{Two Distinct Reservoirs of Water Ice in the Protosolar Nebula}

Formation scenarios of the protosolar nebula (PSN) invoke two main reservoirs of ices present in the disk midplane and that took part in the production of icy planetesimals (see Fig. 7). The first reservoir, located in the inner region of the PSN, contains ices (dominated by $\mathrm{H}_{2} \mathrm{O}, \mathrm{CO}, \mathrm{CO}_{2}, \mathrm{CH}_{4}, \mathrm{~N}_{2}$ and $\mathrm{NH}_{3}$ ) originating from the ISM, which, due to their near solar vicinity, were initially vaporized. The ice vaporization distance never exceeds $\sim 30$ AU from the Sun, depending on the total source luminosity and characteristics of the collapsing cloud (Chick and Cassen 1997). With time, the decrease of temperature and pressure conditions allowed the water in this reservoir to condense at $\sim 150 \mathrm{~K}$ (at typical nebula pressure conditions) in the form of microscopic crystalline ice (Kouchi et al. 1994), leaving negligible water in the vapor phase to condense at lower temperatures where amorphous ice would be expected. It is postulated that a substantial fraction of the volatile species were trapped as clathrates during this condensation phase as long as free water ice was available within $30 \mathrm{AU}$ in the outer solar nebula (Mousis et al. 2000) and there was enough time to overcome the kinetics of clathration. On the other hand, the remaining volatiles that were not enclathrated (due to the lack of available water ice or a low kinetics of clathration) probably formed pure condensates at lower temperatures in this part of the nebula (Mousis et al. 2012a, 2012b). The other reservoir, located at larger heliocentric distances, is composed of ices originating from the ISM that did not vaporize when entering into the disk. In this reservoir, water ice was essentially in the amorphous form and the other volatiles remained trapped in the amorphous matrix (Owen et al. 1999; Notesco and Bar-Nun 2005). 
Interestingly, it has been shown recently that amorphous ice could form from photodesorption and freeze-out of water molecules near the surface layers of the PSN (Ciesla 2014). In these conditions, the transport of the icy grains throughout the outer solar nebula would lead crystalline ice to be lost and reformed as amorphous ice. In turn, amorphous ice generated on the disk's surface could be transformed to crystalline as it migrates through the protoplanetary disk (Ciesla 2014). This cycling scenario needs to be further investigated if one wants to assess its role in the formation or destruction of the two original icy reservoirs. Also, the different transport mechanisms must be simultaneously taken into account at the different stages of the disk's evolution to depict the abundance of water ice in the two reservoirs as a function of the heliocentric distance. For example, as described in Sect. 2.3.2, it has been shown that vertical mixing can decrease the abundance of water inside the $\sim 15 \mathrm{AU}$ of the disk. In contrast, it has also been proposed that the outward vapor diffusion induced by the presence of the iceline with its subsequent condensation in a narrow location (with a width around $0.5 \mathrm{AU}$ ) would deplete the region inside the iceline of vapor down to subsolar values, and increase substantially the ice abundance at the iceline location by factors larger than 10 times the protosolar value (Ali-Dib et al. 2014). In addition, recent works depicting the chemical evolution of both solids and gases from the pre-stellar core to the protostar and circumstellar disk phases (Visser et al. 2009b; Visser et al. 2011; Harsono et al. 2013) should be taken into account to update the representation of the two water ice reservoirs present in the PSN. In these cases, midplane ices may have experienced multiple desorption events during the evolution of the disk. Nevertheless, if one follows this classical picture, icy solids that formed at heliocentric distances less than $\sim 30 \mathrm{AU}$ mainly agglomerated from a mixture of clathrates and pure condensates, whose ratio depends on the amount of available crystalline water and its clathration efficiency (Mousis et al. 2009b). In contrast, solids produced at higher heliocentric distances (i.e., in the cold outer part of the solar nebula) were formed from primordial amorphous ice originating from the ISM. Therefore, depending where the outer solar system bodies formed, clathrates may have been agglomerated in comets (Marboeuf et al. 2010a, 2010b, 2012), and in the building blocks of the giant planets (Gautier et al. 2001; Alibert et al. 2005a, 2005b; Mousis et al. 2012a, 2009b) and in their surrounding satellite systems (Lunine and Stevenson 1985; Mousis 2004; Mousis and Gautier 2004; Mousis et al. 2009a). Regardless the possible presence of clathrates, there is today indirect evidence that comets formed from crystalline water ice because their deuterium-to-hydrogen ratios measured in $\mathrm{H}_{2} \mathrm{O}$ are substantially lower $(\sim 6-12$ times the protosolar value) than the most deuterium-rich primitive meteorite $(\sim 35$ times the protosolar value), whose level of deuteration is expected to be close to the one found in the ice infalling from the presolar cloud (Kavelaars et al. 2011; Hartogh et al. 2011; Ceccarelli et al. 2014). This difference can only been explained by the isotopic exchange between the deuterium-rich water and molecular hydrogen in the gas phase (Kavelaars et al. 2011; Hartogh et al. 2011; Ceccarelli et al. 2014). Hence, in order to enable this reaction, amorphous ice entering the disk from ISM must have been vaporized prior recondensing again in crystalline form, otherwise the isotopic exchange did not occur.

\section{Fractionation in Protostellar Disks}

\subsection{The Deuterium Fractionation: The Link Between Comets and the Earliest Phases of the Solar System and Interstellar Chemistry}

The origin of the volatile ices found in comets is a central issue for understanding the formation and early evolution of the solar system. There are three possibilities. First, one view 
is that all the ices are comprised of pristine interstellar molecules (e.g. Irvine et al. 1996). There is indeed a strong similarity with the many known interstellar molecules believed to form on interstellar grains, although there are differences in the relative abundances of the volatile ices (Mumma and Charnley 2011). Until recently this idea was widely considered to be untenable (see Sect. 3.1.2 and Cleeves et al. 2014a). Second, at the other extreme, it has been advocated that any interstellar molecules experienced total obliteration and 'chemical reset' in the disk (Pontoppidan and Blevins 2014). In this case all the volatile molecules observed in comets must have been formed by nebular chemistry.

Comets and meteorites contain both high-temperature processed materials, such as crystalline silicates, and matter that apparently formed at very low temperatures, as indicated by the isotopic enrichments measured in D and ${ }^{15} \mathrm{~N}$ (Mumma and Charnley 2011). These characteristics are most naturally explained by the large-scale turbulent transport of dust and gas from different nebular chemical environments into the comet-formation zones (Ciesla and Charnley 2006). The recognition that non-equilibrium models of nebular chemistry are essential, particularly in the comet-forming regions, has motivated many detailed studies of protoplanetary disk chemistry, as recently reviewed by Henning and Semenov (2013) (see also Sect. 2.3.2). Turbulence leads to an outward radial diffusion of material from the hot inner nebula and an inwards advection of material from the cooler outer nebula. It also leads to vertical mixing and thus to midplane material experiencing the enhanced UV and X-ray fluxes of the upper disk layers (Glassgold et al. 1997). Hence, in this third partially-mixed scenario some specific volatile molecules and isotopic ratios could be remnant interstellar matter, or derive from warm and cold regions of the nebula (as reviewed by Pontoppidan et al. 2014). Deciding between such molecular origins has been problematic.

\subsubsection{The Molecular Deuteration Process}

Molecular deuteration provides a potential clue to the history of cometary volatiles. Although deuterium in the Universe is a tiny fraction with respect to the hydrogen $(\mathrm{D} / \mathrm{H}=$ $[2.535 \pm 0.05] \times 10^{-5}$ : Pettini and Cooke 2012), its abundance in interstellar molecules has an enormous diagnostic power, because it may be much larger that the elemental D/H ratio. This generally occurs in cold $(\leq 30-50 \mathrm{~K})$ environments and in trace species, namely molecules with abundances smaller than $\sim 10^{-5}$.

The first key point to keep in mind is that, in cold gas, the major deuterium reservoir is HD and only a small fraction of deuterium is locked in other species. An exception to this rule is represented by the water, whose abundance can reach $\sim 10^{-4}$ and, consequently, water might trap a substantial fraction of deuterium.

The second key point is that the deuterium atoms are "extracted" from the HD molecules because the cosmic rays that hit $\mathrm{H}$ and $\mathrm{H}_{2}$ create $\mathrm{H}_{3}^{+}$ions, which in turn react with $\mathrm{HD}$ and form $\mathrm{H}_{2} D^{+}$ions.

$$
\mathrm{H}_{3}^{+}+\mathrm{HD} \rightleftharpoons \mathrm{H}_{2} \mathrm{D}^{+}+\mathrm{H}_{2}+220 \mathrm{~K}
$$

(see also Fig. 6). The reverse reaction has a small energy barrier, which cannot be overcome at low $(\leq 20-30 \mathrm{~K})$ temperatures, so that the $\mathrm{H}_{2} \mathrm{D}^{+} / \mathrm{H}_{3}^{+}$abundance ratio becomes larger than the $\mathrm{D} / \mathrm{H}$ elemental ratio. The kinetics of Reaction (1) at low temperatures is also strongly influenced by the spin state of the reacting hydrogen molecules, i.e., whether they are predominantly in the ortho or para states. The higher internal energy of ortho- $\mathrm{H}_{2}$ means that the reverse process can be driven efficiently if the $\mathrm{H}_{2}$ ortho/para ratio is large. Thus, ortho- $\mathrm{H}_{2}$ acts as a "poison" for interstellar deuteration which requires that most of the $\mathrm{H}_{2}$ be converted to para- $\mathrm{H}_{2}$ (see Pagani et al. 2011). Reaction (1) is the first step towards the 


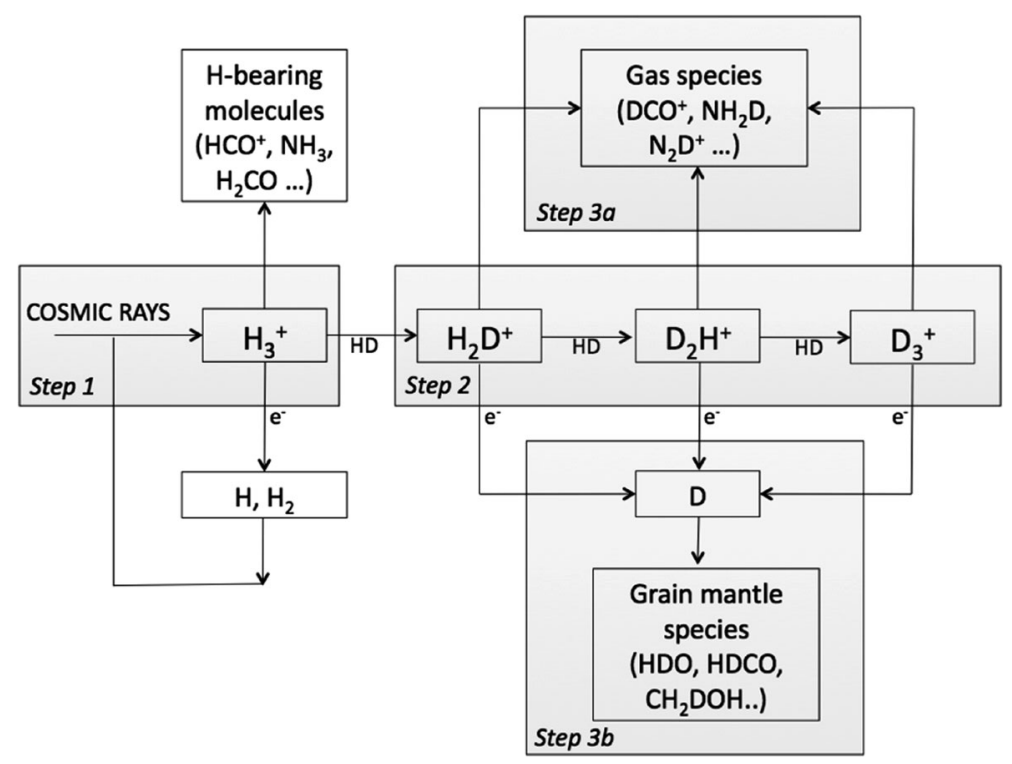

Fig. 8 Schema of the molecular deuteration process in cold gas (Ceccarelli et al. 2014). Molecular deuterium occurs through three basic steps: 1) formation of $\mathrm{H}_{3}^{+}$ions from the interaction of cosmic rays with $\mathrm{H}_{\text {and }} \mathrm{H}_{2}$; 2) formation of $\mathrm{H}_{2} \mathrm{D}^{+}\left(\mathrm{HD}_{2}^{+}\right.$and $\left.\mathrm{D}_{3}^{+}\right)$from the reaction of $\mathrm{H}_{3}^{+}\left(\mathrm{H}_{2} \mathrm{D}^{+}\right.$and $\left.\mathrm{HD}_{2}^{+}\right)$with $\left.\mathrm{HD} ; 3\right)$ formation of other D-bearing molecules from reactions with $\mathrm{H}_{2} \mathrm{D}^{+}\left(\mathrm{HD}_{2}^{+}\right.$and $\left.\mathrm{D}_{3}^{+}\right)$in the gas phase (Step 3a) and on the grain mantles (Step 3b)

deuteration of the trace species, as $\mathrm{H}_{2} \mathrm{D}^{+}$reacts with all neutral molecules and passes, once every three times, the $\mathrm{D}$ atom to the product species. This is schematically shown in Fig. 8. A similar process can also occur through the $\mathrm{CH}_{3}^{+}$ions at slightly higher temperatures.

In practice, therefore, a molecular deuteration enhanced with respect to the $\mathrm{D} / \mathrm{H}$ elemental abundance is a thermometer of the gas temperature.

The third and final key point to keep in mind is that neutral molecules can freeze-out into the grain mantles and, in this way, they can be transmitted through the different stages of the star and planet formation. A sketch of this overall process is shown in Fig. 9. Therefore, ice species formed during the very first phases might be conserved and "reappear" in the gas at much later stages: for example when comets and planets are formed. Conversely, the molecular deuteration in comets and planets can help us to understand in what epoch the relevant species was formed, at least in principle. In this sense, molecular deuteration can be thought of as an Ariadne's thread which links the various phases of solar system evolution. For a more detailed review see Ceccarelli et al. (2014).

\subsubsection{Deuteration in the Solar Nebula}

Deuteration in disks is driven mainly via the reaction of $\mathrm{H}_{2} \mathrm{D}^{+}$ions (Reaction (1)). At low temperatures the reverse process is inhibited and this leads to enhanced deuteration in gaseous molecules through ion-molecule isotope exchange reactions. Elevated atomic $\mathrm{D} / \mathrm{H}$ ratios also pertain and lead to significant deuteration in grain-surface reactions, such as those depicted in Fig. 10 and in the surface reduction of accreted $\mathrm{O}$ atoms to form HDO. Condensation of $\mathrm{CO}$ leads to enhanced abundances of gaseous $\mathrm{H}_{2} \mathrm{D}^{+}$and to significant deuterium fractionation beyond the $\mathrm{CO}$ iceline, as traced by $\mathrm{DCO}^{+}$(Mathews et al. 2013). Enhanced 


\section{FROM A DIFFUSE CLOUD TO A SUN + PLANETARY SYSTEM THE DEUTERIUM FRACTIONATION HISTORY}

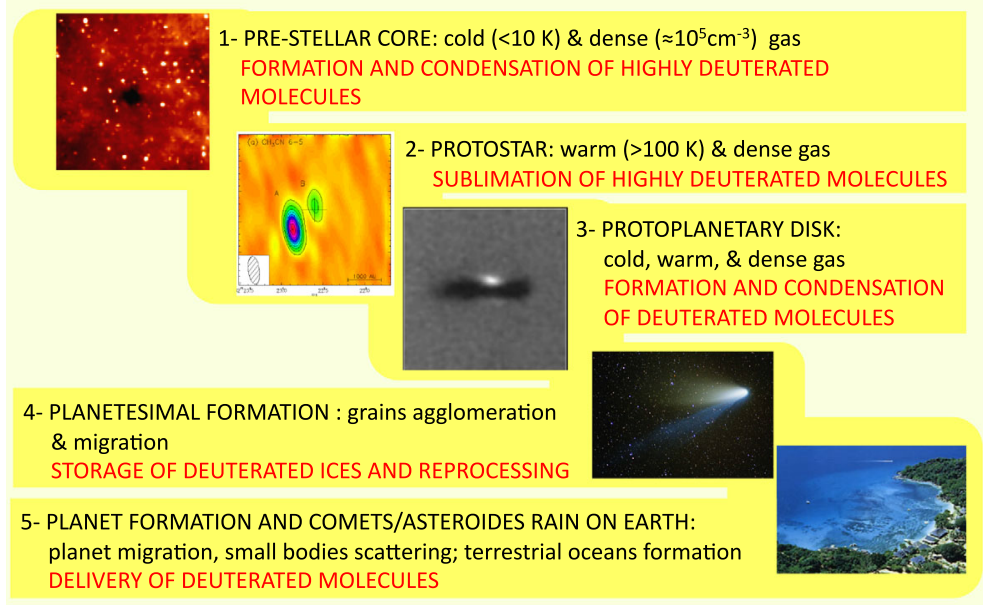

Fig. 9 Schematic summary of the different phases of the formation of a solar-type star and planets, highlighting the formation and fate of deuterated molecules (adapted from Ceccarelli et al. 2014). Likely, the same process gave birth to the solar system

$\mathrm{D} / \mathrm{H}$ ratios in simple molecules, such as water, ammonia and methane, can be produced both in ion-molecule reactions and grain-surface additions of $\mathrm{H}$ and $\mathrm{D}$ to accreted atoms (Ceccarelli et al. 2014). At the CO iceline the grain-surface chemistry depicted in Fig. 10 can lead to very high D/H ratios and multiply deuterated molecules (Charnley et al. 1997; Ceccarelli et al. 2014). Observations show that simple deuterated species like DCN and by $\mathrm{DCO}^{+}$are present in disks (Qi et al. 2008) and we would expect that more complex fractionated organics, e.g., $\mathrm{HDCO}, \mathrm{D}_{2} \mathrm{CO}, \mathrm{CH}_{3} \mathrm{OD}$ and $\mathrm{CH}_{2} \mathrm{DOH}$, to also be present. Between the $\mathrm{CO}$ and $\mathrm{CO}_{2}$ icelines (see Sect. 2.4), large deuterium enrichments can persist in organic molecules since ion-molecule deuteration involving $\mathrm{C}_{2} \mathrm{HD}^{+}$and $\mathrm{CH}_{2} \mathrm{D}^{+}$can persist up to higher temperatures $(\sim 70 \mathrm{~K}$ and $\sim 50 \mathrm{~K})$ than $\mathrm{H}_{2} \mathrm{D}^{+}(\sim 30 \mathrm{~K})$ (Millar et al. 1989). It is possible that the $\mathrm{D} / \mathrm{H}$ ratios of several organic molecules can become re-fractionated even in the warm inner nebula, prior to the condensation of acetylene and methane, both of which will occur outside the $\mathrm{CO}_{2}$ iceline. This means that deuteration can still occur in cold gas that is mixed radially inwards, and be initiated in warmer gas coming from the inner nebula (Willacy 2007; Albertsson et al. 2014).

However, the assumption of interstellar rates of cosmic-ray driven ionization $(\zeta)$ in the cold regions of the outer midplane has recently been called into question. Cleeves et al. (2013) showed that the effects of either deflection by stellar winds or magnetic field topology (through the magnetic mirror effect) could significantly attenuate the penetration of cosmic rays into magnetized protoplanetary disks, and substantially reduce $\zeta$ by several orders of magnitude at the midplane. In this picture the only sources of ionization close to the midplane are scattered X-rays and the decay of short-lived radionuclides. In fact, Dolginov and Stepinski (1994) had previously pointed out that inhomogenous random magnetic fields could also significantly inhibit the transport of cosmic rays throughout the disk and lead to very low values of $\zeta$.

Cleeves et al. (2014a) developed this scenario further by pointing out that effectively extinguishing cosmic ray ionization from the nebular midplane would greatly reduce the 


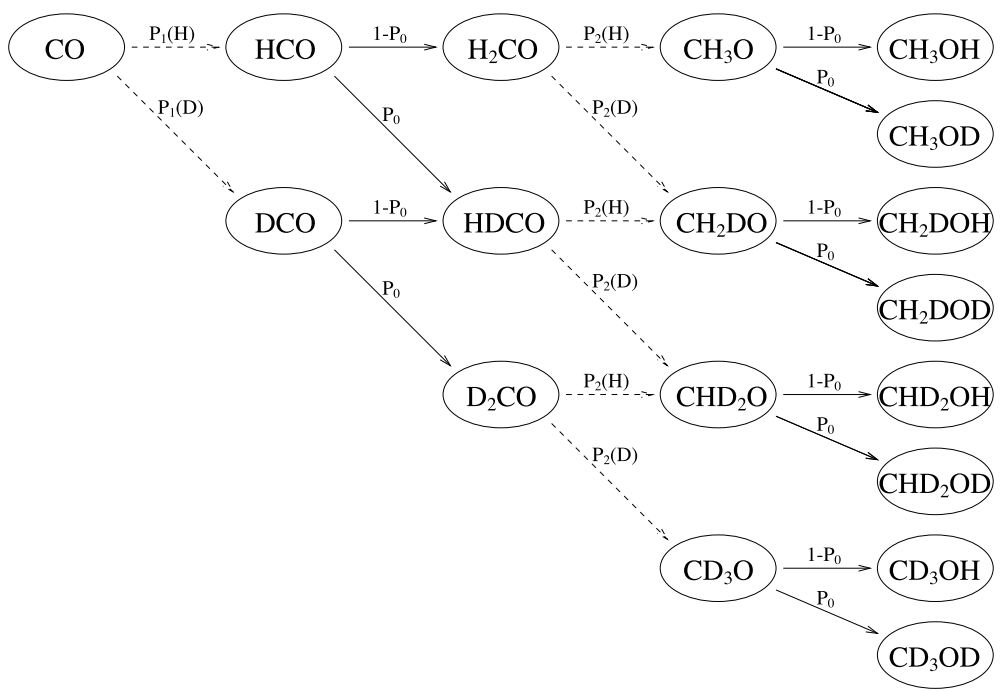

Fig. 10 Grain surface deuteration pathways associated with methanol formation from hydrogenation of $\mathrm{CO}$ ice. The probabilities for each reaction pathway, $\mathrm{P}_{i}$, assume a statistical steady state and are related to the atomic $\mathrm{D} / \mathrm{H}$ ratio of the accreting gas and the energy barrier for addition of an $\mathrm{H}$ or $\mathrm{D}$ atom (from Charnley et al. 1997)

efficacy of Reaction (1) and consequently the generation of enhanced molecular D/H ratios by nebular ion-molecule chemistry. They demonstrated that if the enhanced interstellar $\mathrm{HDO} / \mathrm{H}_{2} \mathrm{O}$ ratios initially available were lost, then nebular chemistry could not regenerate those currently measured in the solar system, concluding that as much as $50 \%$ of the Earth's oceans and perhaps all cometary water is of interstellar origin. Low values of $\zeta$ will also reduce the atomic $\mathrm{D} / \mathrm{H}$ ratio in the disk midplane and so reduce the $\mathrm{D} / \mathrm{H}$ ratios and the level of multiple deuteration in the molecules formed in the scheme of Fig. 10. This may account for the upper limits on $\mathrm{HDCO}, \mathrm{CH}_{2} \mathrm{DOH}$ and $\mathrm{CH}_{3} \mathrm{DOD}$ in comet Hale-Bopp (Crovisier et al. 2004) relative to the large observed interstellar D/H ratios measured in these molecules, which could have been lost or modified upon incorporation into the nebula. Although these molecules could be partially reformed in the cold outer nebula, this process, and any related deuteration, may be less efficient than in molecular clouds. Cometary $\mathrm{CH}_{3} \mathrm{OH} / \mathrm{H}_{2} \mathrm{O}$ ratios are typically $\sim 1-4 \%$, whereas $\sim 5-15 \%$ are typical for the ISM, and can be as high as $40 \%$ (Mumma and Charnley 2011). Cleeves et al. (2014b) also modeled the general protoplanetary disk chemistry for a variety of assumed $\zeta$ values and identified molecular ions that may eventually allow the ionization structure to be determined. If these spatial distributions are observable with ALMA they could provide valuable information on the actual role of cosmic rays in nebular chemistry.

\subsubsection{The Message from Molecular Deuteration}

Figure 11 shows the molecular deuteration in the different objects of the solar system and in objects which are believed to eventually form a solar-like system. It gives us the following major messages:

- All reported solar system objects, including Earth, possess a molecular deuteration larger, by more than a factor 10 , than the $\mathrm{D} / \mathrm{H}$ elemental ratio of the environment where the Sun 
Fig. $11 \mathrm{D} / \mathrm{H}$ ratio in molecules observed in solar system objects and in objects which are believed to eventually form a solar-like system. The filled symbols refer to water measures whereas open symbols refer to organic matter. The different objects are marked (note: CC means Carbonaceous Chondrites, IOM Insoluble Organic Matter, and SOM Soluble Organic Matter). References to the various measures can be found in Ceccarelli et al. (2014)

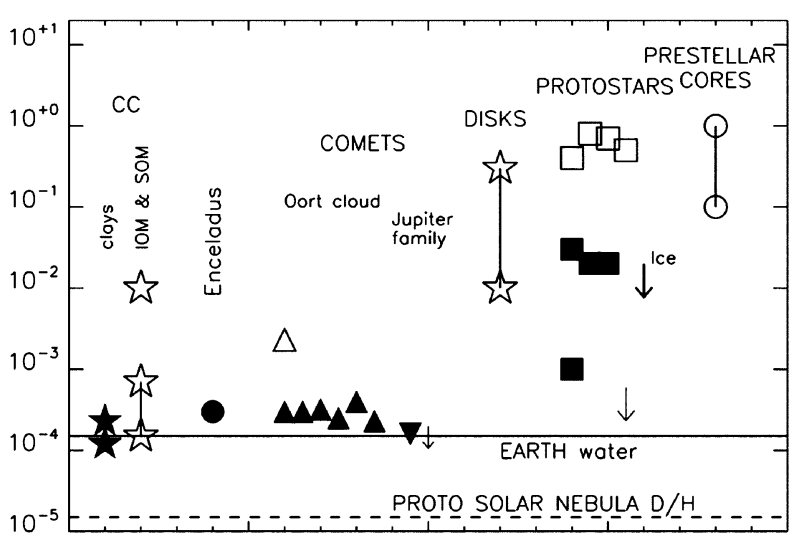

was born, and that is represented by the line marked with "Proto Solar Nebula". This implies that the process of molecular deuteration was at work and it was at least partially inherited from the earlier phases of the solar system formation era.

- There is a general trend, with the objects that formed earlier being also the ones with the largest molecular deuteration, in agreement with the scenario that the process started in very cold conditions. This implies that there was only a partial transmission between the subsequent steps of Fig. 9. Besides, earlier objects in the plot also correspond to material whose distance from the central heating source is larger. Therefore, the trend also tells us that there was not a general and substantial remixing of the different regions (outer and inner, where molecular deuteration is different because of the different temperatures). Exactly how much material was passed through each step or remixed remains, however, unanswered.

- The organic matter is systematically more enriched in deuterium atoms than the water. This is true in protostellar objects, comets and meteorites. However, while this result is robust for the protostellar objects and meteorites, where several measurements of the deuteration have been made, it is less certain for the comets, where so far the only organic species for which molecular deuteration has been measured is HCN. The reason for this dichotomy in the molecular deuteration is completely unclear. In protostellar objects, it is believed that this reflects the different phases of formation of water and organics, with water being formed very early when the gas is not very cold, while the organics were synthesized later from frozen CO. It is possible that a similar process is at the origin of the different deuteration in meteorites and comets. Alternatively, they may conserve a "core" of the protostellar molecular deuteration.

- Molecular deuteration in a given molecule has a strong temperature dependence at low temperatures. This is true for both gas phase and icy grain surface formation. In ice mantles the higher binding energy of deuteration atoms at $<20 \mathrm{~K}$ leads to significant enhancements of deuterated species that form via hydrogenation reactions (see also Fig. 10). These low temperatures correspond to what modelers predict for the cold midplane in the giant planet formation zone of the protoplanetary disk. Measurements of the molecular deuteration in comets provide a potential tracer of the thermal conditions under which they formed. For further discussion see Bockelée-Morvan et al. (2015, this issue).

- Molecular deuteration in a given molecule has a strong temperature dependence at low temperatures. This is true for both gas phase and icy grain surface formation. In ice mantles the higher binding energy of deuteration atoms at $<20 \mathrm{~K}$ leads to significant enhance- 


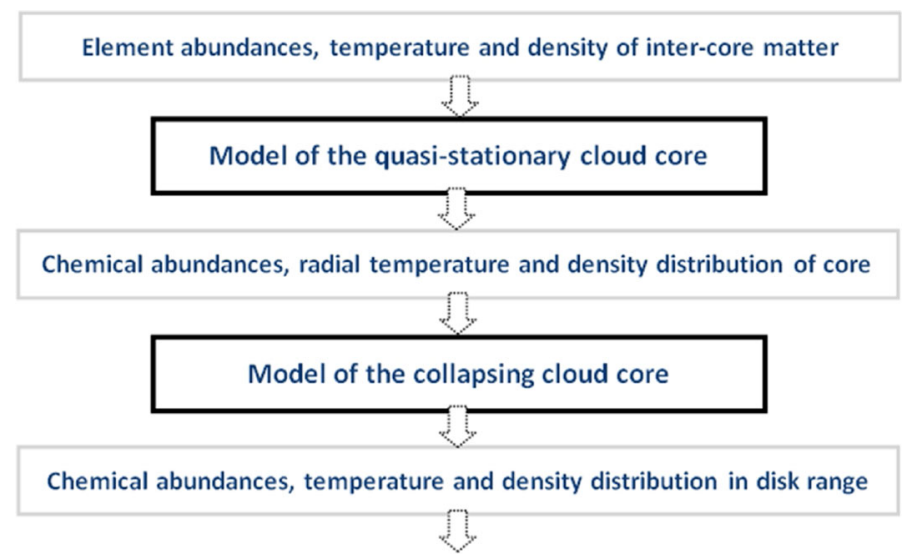

Fig. 12 Flowchart illustrating the evolution of the two precursor stages (the quasi-stationary cloud core and the collapsing core) to the solar nebula. Evolution in these stages will affect the composition of material incorporated into the solar nebula and hence the composition of planetary bodies that form within it

ments of deuterated species that form via hydrogenation reactions (see also Fig. 10). Measurements of the molecular deuteration in comets provide a potential tracer of the thermal conditions under which they formed. For further discussion see Bockelée-Morvan et al. (2015, this issue).

\subsubsection{A Model of the Deuteration of Water in the Early Stages of the Evolution of the Solar Nebula}

Molecular deuteration potentially provides a means of tracing the history of solar system material and its links to the parent molecular cloud. In this section we present the results of a specific model of the evolution of the deuteration of water during the collapse of the parent molecular cloud, since the composition of the protosolar nebula depends in part on the chemical history of this core (Fig. 12). The evolution of the core has been modeled recently by Aikawa et al. (2012); Wakelam et al. (2012); Taquet et al. (2014) and Tornow et al. (2014a, 2014b).

In these models the cloud core is initially described as a Bonner-Ebert sphere (Aikawa et al. 2008) which is embedded in a constant density gas-shell with a visual extinction of up to 4 magnitudes (Wakelam et al. 2012), and irradiated by an external UV field. It is allowed to evolve for between 1 and 6 Myrs (Tornow et al. 2014a). Both the gas phase ${ }^{2}$ and grain $^{3}$ chemistry is followed. Since the core is only partially shielded, photo-chemistry has a clear effect on the radial profile of the relative water abundance in the outer core region (Fig. 13). At the end of the quasi-stationary core stage the temperature of the gas and dust decreases to 8-9 $\mathrm{K}$ in the center for boundary values between 13-15 K. Consequently, water ice builds up on the cold grains, reaching to a fairly constant relative abundance of $\sim 10^{-4}$ within $10^{5}-10^{6}$ years. In contrast, Fig. 13 shows a clear radial gradient for the gas phase water abundance. Relatively low ratios (about $0.01-10 \mathrm{ppm}$ ) of gas to dust phase abundance were obtained for the outer core range with up to $25 \mathrm{ppm}$ in the inner region. Caselli et al. (2012)

\footnotetext{
${ }^{2}$ Nahoon_public_apr11.tar.gz at http://kida.obs.u-bordeaux1.fr/models/.

3 disk_chemistry_OSU08ggs_UV.zip at http://www.mpia.de/homes/semenov.
} 

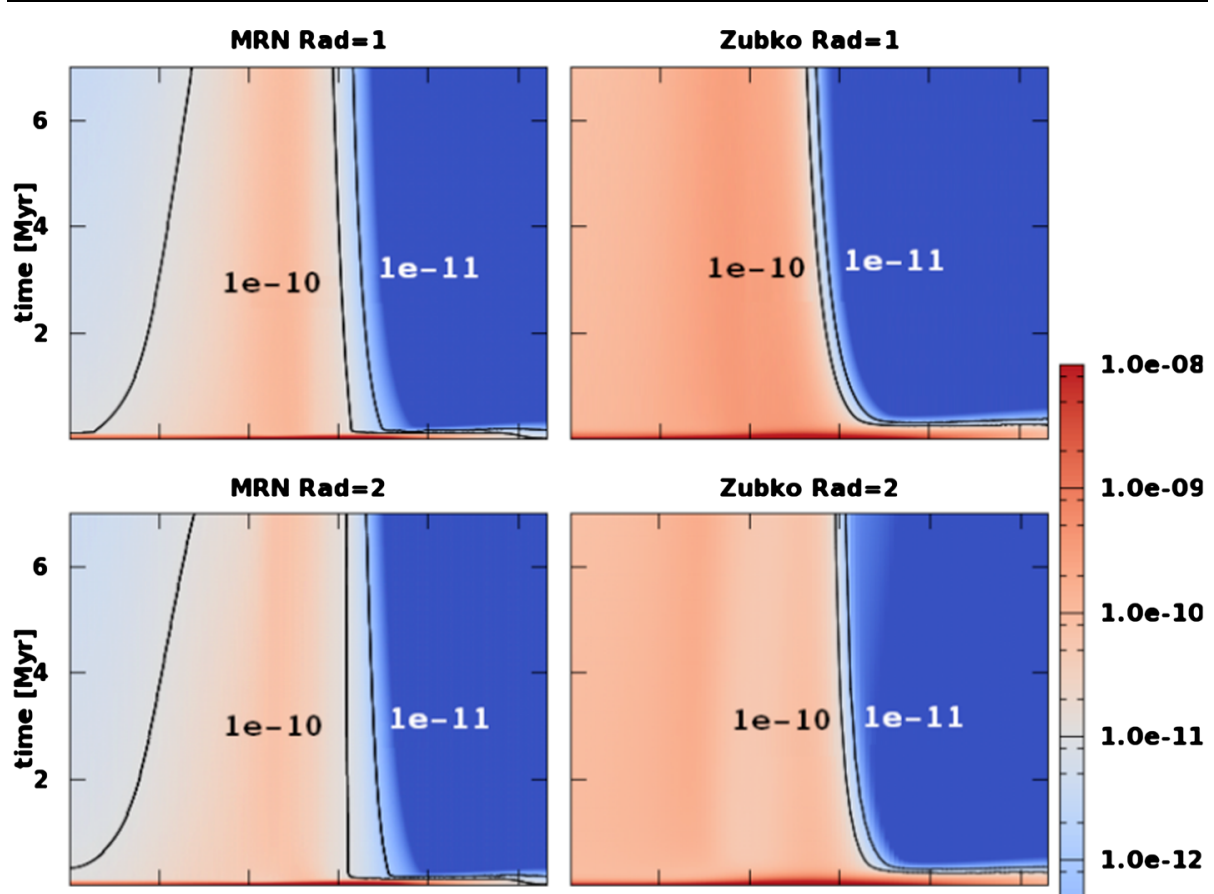

\section{0e-09}

$1.0 \mathrm{e}-10$

$1.0 \mathrm{e}-11$
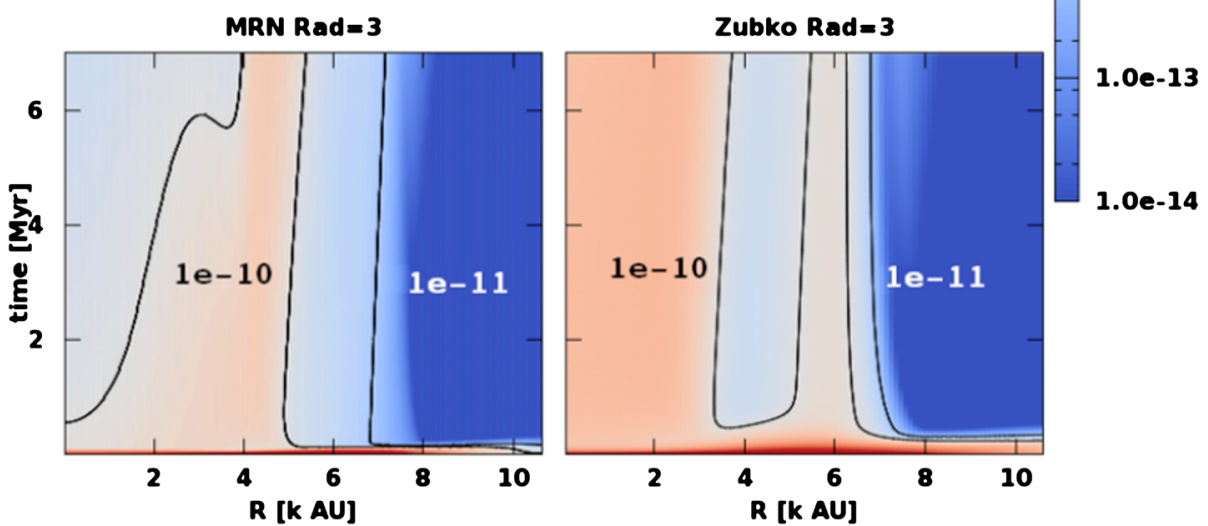

Fig. 13 Relative gas phase abundance of water versus time and radius $\left(1 \mathrm{kAU}=10^{3} \mathrm{AU}\right)$. The intensity of the standard interstellar radiation field (IRSF) is scaled by $\operatorname{Rad}=1,2,3$. Two grain distributions are used. MRN refers to Mathis et al. (1977) and Zubko refers to Zubko et al. (2004). The mean grain size is $5.5 \times 10^{-5} \mathrm{~cm}$ and $3.5 \times 10^{-5} \mathrm{~cm}$ for MRN and Zubko respectively. The cosmic ray ionization rate is taken to be $10^{-17} \mathrm{~s}^{-1}$. The related gas and grain chemistry is modeled using a combined code (Tornow et al. 2014a)

determined higher ratios due to a higher efficiency of water photodesorption; a consequence of the larger grain size and smaller photodesorption yield used in the Tornow et al. models. If the deuterium chemistry is included according to the method of Albertsson et al. (2013) the temporal and radial distribution of the gas phase $\mathrm{HDO} / \mathrm{H}_{2} \mathrm{O}$ ratio can be derived. It reaches a maximum of about 0.02 in the medium shielded core region, and a minimum of $10^{-4}$ in the low shielded outer boundary region (Fig. 14). In contrast, the dust phase ratio of $\mathrm{HDO} / \mathrm{H}_{2} \mathrm{O}$ 


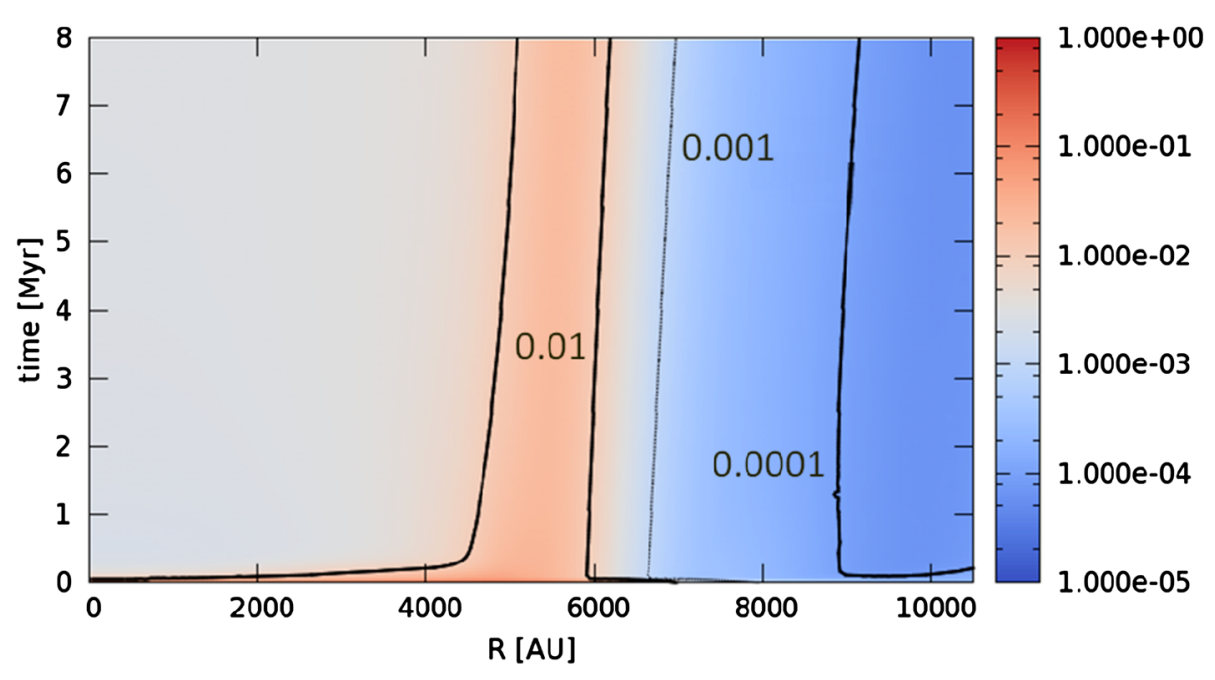

Fig. 14 Temporal and radial $\mathrm{HDO} / \mathrm{H}_{2} \mathrm{O}$ distribution in the gas phase (Tornow et al. 2014b) for the standard ISRF and MRN grain size (see Fig. 13)

is clearly larger and only varies between 0.01 and 0.1 . Its maximum is located in the well shielded inner core region at very early times $\left(\sim(2-3) \times 10^{5}\right.$ years $)$.

The collapse of the cloud core forms a protostar surrounded by an optically thick disk and an outer envelope (Saigo et al. 2008; Schönke and Tscharnuter 2011). During this stage the chemical evolution can be followed along a Lagrangian path from the outer core boundary to the center of the core (Tornow et al. 2014b). Figure 15 shows the $\mathrm{HDO} / \mathrm{H}_{2} \mathrm{O}$ ratio along each member of the Lagrangian path set. Water formed in the quasi-stationary core stage is transported into the region of terrestrial planet formation. Signatures of large-scale, supersonic water inflows have been seen with Herschel (Mottram et al. 2013) who determined that these flows occur on the core scale ( $\leq 10^{4}$ AU), e.g., IRAS 15398 and L1527 or at least on the envelope scale ( $\geq 3 \times 10^{4}$ AU), e.g., NGC1333-IRAS4A. Based on their data Mottram et al. suggest an outside-in collapse, in agreement with the model shown in Fig. 15.

The gas phase deuteration of water varies widely, depending on location and evolutionary time. At $1 \mathrm{AU}$ it ranges from 0.001 for $0.1-0.2 \mathrm{Myrs}$, to 0.1 for $0.3 \mathrm{Myrs}$ and to 0.03 for times later than 0.4 Myrs. A minimum $\mathrm{HDO} / \mathrm{H}_{2} \mathrm{O}$ ratio near 0.001 extends from the radial region around $0.1 \mathrm{AU}$ at early collapse times to nearly $9 \mathrm{AU}$ at later times. The predicted $\mathrm{HDO} / \mathrm{H}_{2} \mathrm{O}$ ratios of Wakelam et al. (2012) and Tornow et al. (2014b) are slightly lower than those of Aikawa et al. (2012) but larger than the ratios observed by Persson et al. (2014). An over-estimation of the water deuteration may be caused by a low collapse temperature which would suggest perfect cooling. Including the ortho-to-para ratio of $\mathrm{H}_{2}$ can also lead to lower $\mathrm{HDO} / \mathrm{H}_{2} \mathrm{O}$ ratios (Taquet et al. 2013, 2014). In addition, water formed during the quasi-stationary core stage must be destroyed by the UV radiation of the interstellar radiation field, nearby stellar sources or X-rays (producing secondary UV photons in a similar way as cosmic rays) and UV photons from the protostar (France et al. 2014). As a result, photodissociation of deuterium-rich water molecules $(\mathrm{HDO}+h v \longrightarrow \mathrm{D}+\mathrm{OH}$, or $\mathrm{H}+\mathrm{OD})$, together with 1-D radial or vertical mixing enable a subsequent reformation of water by hot neutral reactions in the inner disk plane (Furuya et al. 2013). 


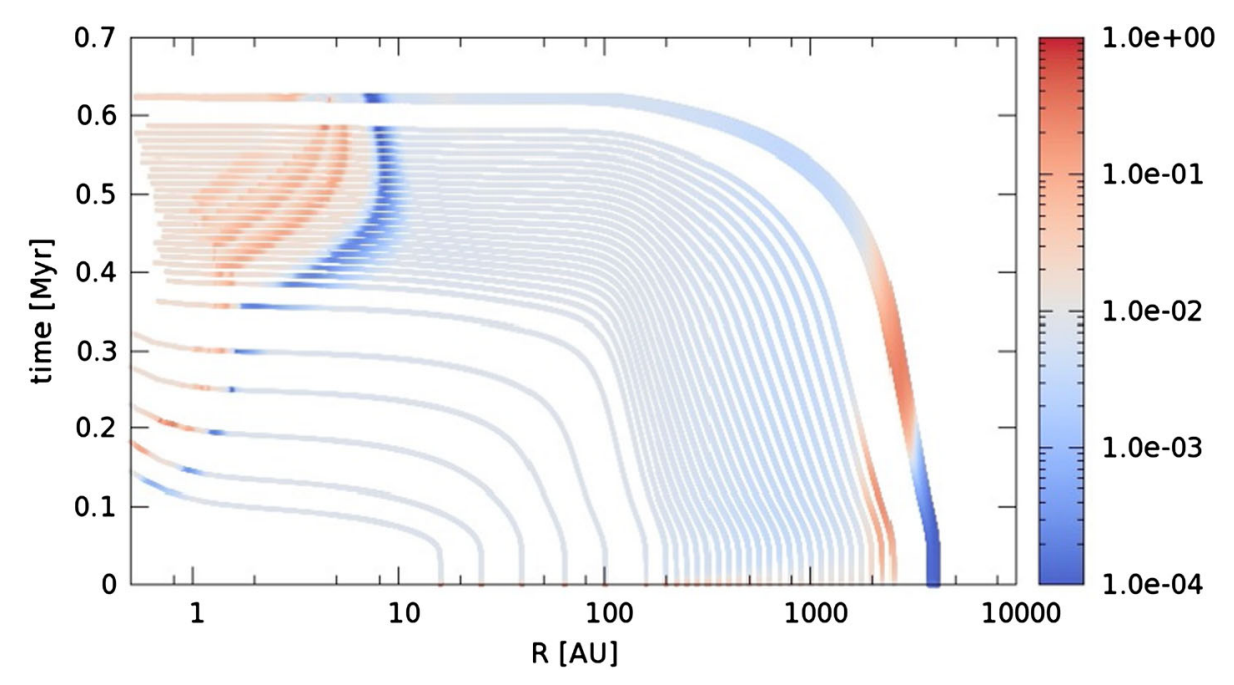

Fig. $15 \mathrm{HDO} / \mathrm{H}_{2} \mathrm{O}$ ratio in the gas phase on Lagrangian paths. At $1 \mathrm{AU}$ the $\mathrm{HDO} / \mathrm{H}_{2} \mathrm{O}$ ratio varies between $10^{-3}$ and 0.1 , while in the dust phase it is much smoother, and only varies between 0.03 and 0.05 (Tornow et al. 2014a, 2014b)

\subsection{Fractionation of Carbon and Nitrogen in the Solar Nebula}

Isotopic enrichment of ${ }^{15} \mathrm{~N}$ in cometary organics (the nitriles $\mathrm{CN}$ and $\mathrm{HCN}$ ) and ammonia is commonly observed (Jehin et al. 2009; Bockelée-Morvan et al. 2015, this issue). Chemical models have shown that fractionation in low-temperature ion-molecule reactions can produce large ${ }^{15} \mathrm{~N}$ enrichments in interstellar clouds (Rodgers and Charnley 2008; Wirström et al. 2012). However, a recent study by Roueff et al. (2015) has demonstrated that several key reactions may in fact possess energy barriers, leading to doubts as the efficacy of ion-molecule processes in $\mathrm{N}$ fractionation. Of particular interest for comets is that ${ }^{15} \mathrm{~N}$ fractionation in the outer nebula should be similarly impaired by low values of the cosmic ray ionization rate, $\zeta$. Thus, as found by Cleeves et al. (2014a) for water deuteration, the low ${ }^{14} \mathrm{~N} /{ }^{15} \mathrm{~N}$ ratios commonly measured in both Oort Cloud and Jupiter Family comets is strongly suggestive of an interstellar origin.

The ${ }^{12} \mathrm{C} /{ }^{13} \mathrm{C}$ ratios measured thus far in simple cometary molecules $(\mathrm{CO}, \mathrm{HCN}, \mathrm{CN})$ are solar (Jehin et al. 2009) and Bockelee-Morvan (these proceedings). In regions of the nebula where water has condensed, the gaseous $\mathrm{C} / \mathrm{O}$ elemental ratio can increase to order unity (Öberg et al. 2011). Because water, $\mathrm{OH}$ and $\mathrm{O}_{2}$ recycle carbon nuclei back into $\mathrm{CO}$ (Langer et al. 1984), ion-molecule fractionation in regions with $\mathrm{C} / \mathrm{O} \sim 1$ will act instead to channel carbon nuclei into simple organic molecules. Thus, as the sole carbon fractionation process involves the ${ }^{13} \mathrm{C}^{+}$exchange reaction with ${ }^{12} \mathrm{CO}$ (Langer et al. 1984), CO condensation may in fact be the reason for the almost uniformity of cometary ${ }^{12} \mathrm{C} /{ }^{13} \mathrm{C}$ ratios measured to date. On the other hand, the condensed $\mathrm{CO}$ is predicted to produce distinctive ${ }^{12} \mathrm{C} /{ }^{13} \mathrm{C}$ fractionation patterns in the organic molecules formed in the grain-surface reaction scheme of Fig. 6 (Charnley et al. 2004). These involve molecules whose ${ }^{13} \mathrm{C}$ isotopologues have not yet been detected in comets (e.g., $\mathrm{H}_{2} \mathrm{CO}, \mathrm{CH}_{3} \mathrm{OH}$ ) but for which the predicted fractionation has been confirmed by observations of protostellar envelopes (Wirström et al. 2011). In this case, once again, ion-molecule pathways to ${ }^{13} \mathrm{C}$ fractionation in the nebula are predicated on the presence of significant flux of cosmic rays. 
If ion-molecule reactions indeed play a greatly reduced role in the cooler parts of the disk then the major sources of nebular fractionation will involve UV photochemistry. Isotope selective photodissociation of $\mathrm{CO}$ has been proposed as an explanation of the oxygen isotope ratios measured in meteorites (Lyons and Young 2005). Isotopic enrichment of ${ }^{13} \mathrm{C},{ }^{15} \mathrm{~N}$ and $\mathrm{D}$ though isotope-selective photodissociation of isotopologues of $\mathrm{CO}, \mathrm{N}_{2}$ and HD has also been considered (Woods and Willacy 2009; Heays et al. 2014; Cleeves et al. 2014a). However, these processes will only occur in thin upper layers of the disk and so will be negligible at the midplane. Thus, to be viable, these processes require efficient downward vertical mixing in the disk. On the other hand, ISP occurring in the over layers of the presolar core, where the interstellar UV field is attenuated, could in principle produce isotopic anomalies in the oxygen and carbon isotopes (Yurimoto and Kuramoto 2004; Lee et al. 2008; Charnley and Rodgers 2009). This environment could also ultimately produce significant fractionation in nitrogen-bearing species but this has not yet been demonstrated quantitatively.

\subsection{Solar Nebula Chemistry and the Origin of Oxygen Isotopic Anomalies in the Solar System}

Oxygen is a very common element that is a major constituent of many minerals and rocks. It exists in three stable isotopes: ${ }^{16} \mathrm{O},{ }^{17} \mathrm{O}$ and ${ }^{18} \mathrm{O}$. The fractionation between these isotopes is not consistent across all solar system samples, as might be expected if the oxygen isotopes were homogeneously distributed across the solar nebula, and if only mass-dependent fractionation processes were active. Figure 16 is a three-isotope plot of the oxygen isotopic compositions of primitive solar system materials such as chondrites, chondrules, shows that their relative isotopic compositions are different compared to those of terrestrial samples (McKeegan et al. 2011; Yurimoto et al. 2007; Clayton 1993) The terrestrial line is characteristic of mass-dependent fractionation, where ${ }^{18} \mathrm{O}$ is twice as fractionated as ${ }^{17} \mathrm{O}$ relative to ${ }^{16} \mathrm{O}$, whereas the steeper, slope- 1 line, of the primitive materials suggests it is the result of mass-independent fractionation.

There are three possible mechanisms that could achieve this mass-independent effect (Yurimoto et al. 2007). Firstly the isotope anomalies could represent an inherited heterogeneity in solar system materials, where the solids are rich in ${ }^{16} \mathrm{O}$ and the gas in the rarer isotopes. This could occur either from nucleosynthesis in other stars (Clayton et al. 1973) or from nuclear reactions triggered by energetic particles produced by the Sun or by galactic cosmic rays (Lee 1978). These nuclear processes could produce fluctuations in the ${ }^{16} \mathrm{O}$ abundance. The lack of observed isotopic anomalies in other elements suggests this mechanism is unlikely (Clayton 1993). Additionally, ${ }^{16} \mathrm{O}$ presolar grains are rare in meteorites (Nagashima et al. 2004; Nittler 2003).

A second suggestion is chemical fractionation within the solar nebula. Differences in the reaction rates of symmetric molecules, e.g. ${ }^{16} \mathrm{O}^{16} \mathrm{O}$, compared to asymmetric ones, e.g. ${ }^{16} \mathrm{O}^{17} \mathrm{O}$, can lead to the observed isotopic distributions, since the most abundant isotope is almost entirely contained in the symmetric molecules, while the rarer isotopes are in the asymmetric ones (Heidenreich and Thiemens 1983). However, the exact reactions by which this effect might be achieved are uncertain (Yurimoto et al. 2007).

The final possibility is isotope-selective photodissociation of $\mathrm{CO}$, either in the parent cloud (Yurimoto and Kuramoto 2004) or at the surface of the protosolar disk (Young 2007; Lyons and Young 2005) could also result in enhancements of the rare isotopes of oxygen. CO dissociates when irradiated by far-ultraviolet photons with wavelengths between 


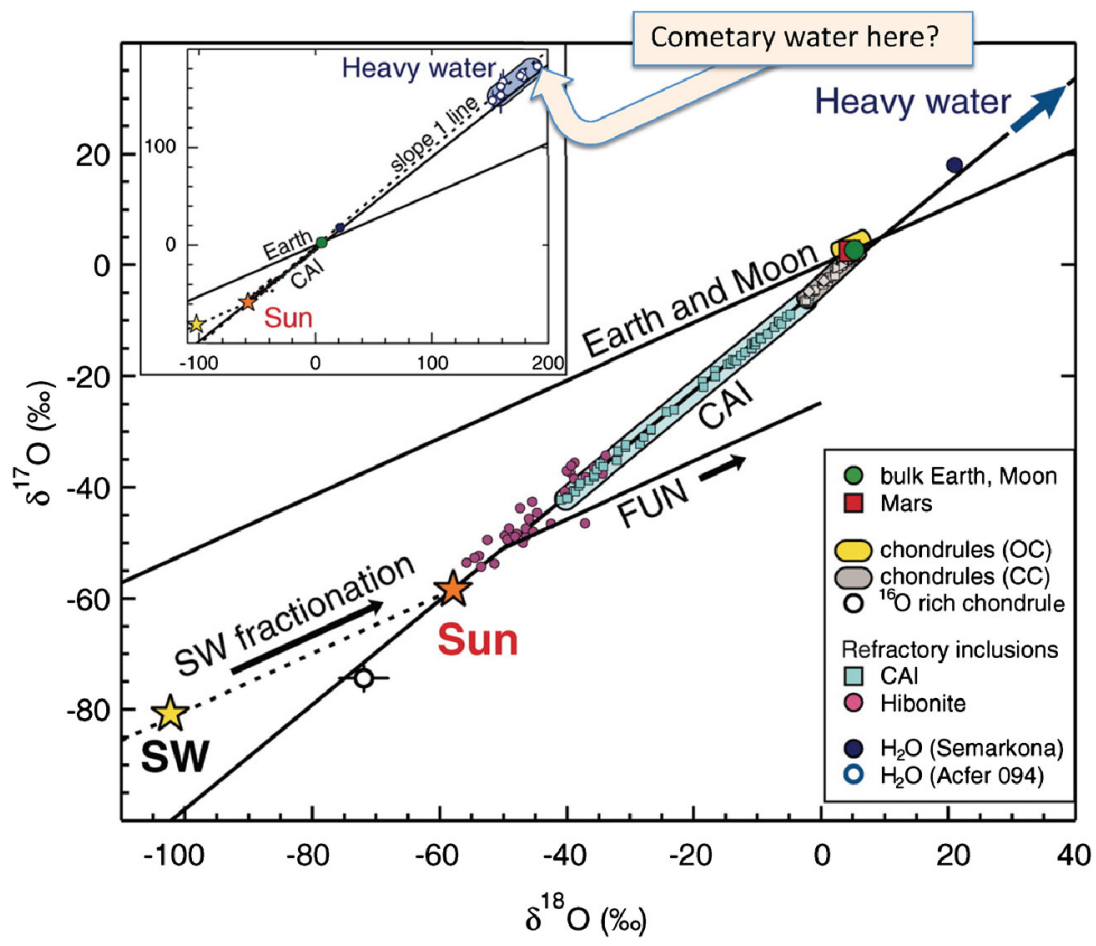

Fig. 16 A three-isotope plot for oxygen in the solar system. The Earth and Moon lie along a fractionation line with slope 0.52 . This line is consistent with fractionation of a single homogenous source, by processes that depend on the mass of the isotopes, so that ${ }^{18} \mathrm{O}$ is nearly twice as fractionated as ${ }^{17} \mathrm{O}$. Data from meteorites and the Genesis mission falls along a slope-1 line indicating they contain material that has undergone some kind of mass-independent fractionation. The Genesis results suggest that much of the rocky material in the inner solar system was enriched in ${ }^{17} \mathrm{O}$ and ${ }^{18} \mathrm{O}$ relative to ${ }^{16} \mathrm{O}$ before the accretion of the first planetesimals. Hence rocky planets are not made of the average nebular material that formed the Sun. From McKeegan et al. (2011) (reprinted with permission)

$91.2 \mathrm{~nm}$ and $110 \mathrm{~nm}$. The dissociation process occurs in two steps, with $\mathrm{CO}$ first entering a bound excited state before dissociating. As a result the $\mathrm{CO}$ absorption spectrum consists of a large number of narrow lines at specific wavelengths determined by the vibrational and rotational levels involved. The change in mass between $\mathrm{C}^{16} \mathrm{O}$ and its isotopologues $\mathrm{C}^{17} \mathrm{O}$ and $\mathrm{C}^{18} \mathrm{O}$ slightly shifts the lines in the absorption spectra, and consequently the spectra for the different isotopologues do not overlap. Hence when $\mathrm{CO}$ is dissociated, either in the protostellar disk or in the molecular cloud core, the different isotopologues do not shield each other (see, for example, Visser et al. 2009a). $\mathrm{C}^{16} \mathrm{O}$ will become optically thick closest to the surface, in a region where $\mathrm{C}^{17} \mathrm{O}$ and $\mathrm{C}^{18} \mathrm{O}$ can still dissociate. When coupled with turbulent mixing that can transport the ${ }^{17} \mathrm{O}$ and ${ }^{18}$ towards the colder, shielded midplane of the disk this can result in the formation of water molecules that are enhanced in the rarer isotopes and which could later be incorporated into CAIs and other meteoritic material.

The in situ measurements that Rosetta will make will directly probe the isotopic composition of the volatile component of a comet. This will test the viability of the potential causes of the oxygen isotopic anomalies and provide new clues as to the origin of the cometary material and the conditions under which it formed. 


\section{The Origin of the Nitrogen Deficiency in Comets}

Nitrogen is one of the most abundant elements in the PSN (Asplund et al. 2009). Its abundance was measured in the giant planets atmospheres in which its stable thermodynamic form is $\mathrm{NH}_{3}$. The nitrogen abundance is found moderately to substantially enriched, compared to its solar value, in Saturn and Jupiter (Mousis et al. 2015), but is loosely constrained in Uranus and Neptune (de Pater et al. 1989; de Pater and Richmond 1989; Ali-Dib et al. 2014). A puzzling revelation was the apparent severe depletion of nitrogen in comets. Mass spectrometry measurements of 1P/Halley's dust composition allowed to find that the nitrogen-to-oxygen ratio is depleted by a factor $\sim 3$ with respect to the solar value (Jessberger and Kissel 1991). Subsequent analysis showed that the ice part of the comet is depleted in nitrogen by a factor up to 75 (Wyckoff et al. 1991). This apparent depletion appears controversial because the $\mathrm{N}_{2}$ molecule, thought to be the main nitrogen-bearing volatile in the whole PSN (Lewis and Prinn 1980; Mousis et al. 2002), has a molecular mass close to that of the very abundant $\mathrm{CO}$ molecule, implying that the mass spectrometer aboard the Giotto spacecraft did not have enough resolution to separate the two molecules (Eberhardt et al. 1987).

More recently, Cochran et al. (2000) used the 2DCoude spectrograph on the 2.7-m Harlan J. Smith telescope of McDonald Observatory to observe the $\mathrm{N}_{2}^{+} / \mathrm{CO}_{2}^{+}$in comets $22 \mathrm{P} / 1995$ $\mathrm{S} 1$ (deVico) and C/1995 O1 (Hale-Bopp), and found values around $\sim 1 \times 10^{-4}$. These values hint at a severe depletion in $\mathrm{N}_{2}$ since the $\mathrm{N}_{2}^{+} / \mathrm{CO}^{+}$ratio is usually equal to $\mathrm{N}_{2} / \mathrm{CO}$. This is surprising specially that $\mathrm{N}_{2}$ and $\mathrm{CO}$ have very close condensation temperatures (Fray and Schmitt 2009). Moreover, efforts were made to find $\mathrm{N}_{2}$ using the Far Ultraviolet Spectroscopic Explorer, but with no success (Bockelée-Morvan et al. 2004). All these observations, and many more, motivated theorists to try to explain this depletion.

In Iro et al. (2003), the authors assumed that volatiles are accreted by comets as clathrates (Lunine and Stevenson 1985), and explain the nitrogen depletion by the low efficiency of $\mathrm{N}_{2}$ trapping in water ices. One of this work's conclusions is that $\mathrm{N}_{2}$ can be trapped only if water ice is present in abundance higher than $2.8 \times$ solar value. This is due to the relative ease by which $\mathrm{CO}$ can be trapped in comparison with $\mathrm{N}_{2}$, so this last can be clathrated only if there is enough water left after all the present $\mathrm{CO}$ was captured. Since $\mathrm{N}_{2}$ is trapped in crystalline water ice at temperatures lower than CO (Mousis et al. 2010), their work implied that comets might have formed at temperatures higher than $45 \mathrm{~K}$, allowing the clathration of $\mathrm{CO}$ but not $\mathrm{N}_{2}$. This temperature lower limit is one of shortcomings of this model since in many protoplanetary disks models, temperature is thought to decrease below this value (Hueso and Guillot 2005). The recent observation of the CO iceline in TW Hya also supports these models (Qi et al. 2013a). Another caveat is the reliability of the Iro et al. (2003)'s determination of the $\mathrm{N}_{2} / \mathrm{CO}$ ratio in clathrates since the potential parameters describing the guest-clathrate interaction in their statistical model correspond to guest-guest interactions (case of pure solutions) and not to the guest-water interactions usually used in such models (Mousis et al. 2012b). Also, the model is unable to explain the thick $\mathrm{N}_{2}$ ice cover on Triton and Pluto, both bodies thought to be formed in the comets region (Lellouch et al. 2011).

Motivated by the shortcomings of Iro et al. (2003), Mousis et al. (2012b) introduced another statistical model based on an appropriate set of potential parameters and that seems to address these issues. In this model, volatiles are also accreted as clathrate-hydrates, except $\mathrm{N}_{2}$ which, due to its poor trapping propensity, is assumed to condense at $\sim 20 \mathrm{~K}$ in the PSN and subsequently trapped in pure crystalline form in comets. Later radiogenic heating by the decay of short-lived nuclides will heat up these bodies to temperatures enough to liberate the trapped $\mathrm{N}_{2}$, but not $\mathrm{CO}$. In Triton and Pluto, the gravity is sufficiently important 
to prevent the gaseous $\mathrm{N}_{2}$ from escaping, and let it condense on the surface and form the observed cover. In the case of comets however, the gravity is negligible and the gaseous $\mathrm{N}_{2}$ will escape, leaving comets depleted in nitrogen. A shortcoming of this model is that no radiogenic particles were found by stardust (Flynn 2008).

A recent model, applied to the description of Uranus and Neptune formations, also provides an alternative solution. Ali-Dib et al. (2014) proposed that the diffusive redistribution of vapor will remove the gaseous $\mathrm{N}_{2}$ from the region below its iceline, possibly explaining the probable nitrogen depletion in Uranus and \& Neptune if they formed in this region. One can invoke a similar argument for the nitrogen depletion in comets. In this case, the CO-rich comets will need to form between the $\mathrm{CO}$ and $\mathrm{N}_{2}$ icelines, in order to accrete $\mathrm{CO}$ in solid form (since its vapor will also be depleted below its iceline) but not $\mathrm{N}_{2}$. The relatively small distance between the two icelines $(\sim 4-5 \mathrm{AU})$ is however a caveat for this model since it imposes the formation of all comets in this restricted area of the outer PSN.

Finally, all these models took the $\mathrm{N}_{2}$ depletion at face value. As discussed above, the $\mathrm{N}_{2}$ molecule's mass is very close to $\mathrm{CO}$, and the two molecules can be distinguished only using an instrument with a very high spectral resolution, such as ROSINA on board the Rosetta spacecraft (Gulkis and Alexander 2008). If this (or another) instrument is able to detect important amounts of $\mathrm{N}_{2}$ in a comet, this might resolve at least part of the problem. Since important chemical differences are known to exist between Oort Cloud comets and Jupiter Family comets (Mumma and Charnley 2011), positive detections or definitive exclusion of $\mathrm{N}_{2}$ in both types of populations are needed before drawing final conclusions.

\section{Spin Temperature as a Cosmogonic Indicator in Comets}

The volatile composition, $\mathrm{D} / \mathrm{H}$ ratio in molecular volatiles and the spin temperature, as given by the ortho-to-para ratio in water or other molecules, are considered to be possible cosmogonic indicators for comets and as such may be interpreted as providing information about the formation history of the molecules in question. In this section, we discuss spin temperature as a possible cosmogonic indicator for comets.

Molecules that contain identical hydrogen nuclei (like $\mathrm{H}_{2} \mathrm{O}$ and $\mathrm{CH}_{4}$ ) display nuclear spin isomers. Water, for example, has two spin species referred to as ortho- $\mathrm{H}_{2} \mathrm{O}$ if the nuclear spins of the hydrogen atoms are parallel and para- $\mathrm{H}_{2}$ if they are anti-parallel. The lowest energy level of para- $\mathrm{H}_{2} \mathrm{O}$ lies $23.8 \mathrm{~cm}^{-1}(\sim 34 \mathrm{~K}$ ) below the lowest ortho level, so when water is formed at temperatures below about $50 \mathrm{~K}$, the formation of para states is preferred and the ortho-para ratio (OPR) is less than the statistical equilibrium value of 3 . Likewise for methane, statistical equilibrium corresponds to abundance ratios A:E:F of 5:2:9 and statistical equilibrium is reached for temperatures above about $60 \mathrm{~K}$.

OPRs have been routinely measured in water in comets since the apparition of comet 1P/Halley (Mumma et al. 1987). This is currently done by fitting synthetic fluorescent emission models independently for each spin state as shown in Figs. 17 and 18. Corresponding spin temperatures are determined by placing the OPR on a theoretical curve such as that for water shown in Fig. 19. OPR is also commonly inferred for ammonia by measuring the OPR for $\mathrm{NH}_{2}$, thought to be formed primarily via photodissociation of ammonia (Kawakita et al. 2004; Shinnaka et al. 2011). More recently, spin temperature has been measured in cometary methane for comets C/2007 N3 Lulin (Gibb et al. 2012), C/2007 W1 (Villanueva et al. 2011), C/2004 Q2 (Bonev et al. 2009; Kawakita and Kobayashi 2009), and C/2001 Q4 (Kawakita et al. 2005). 


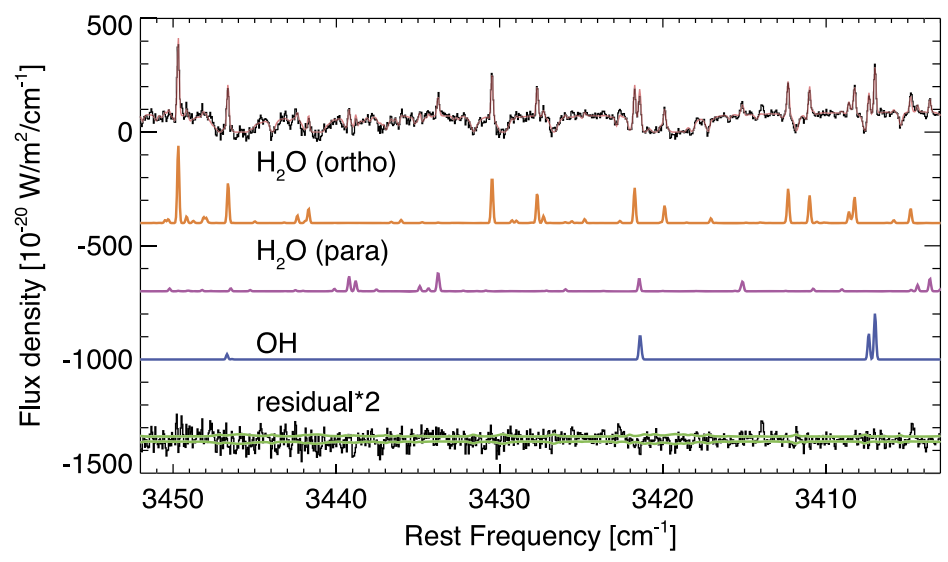

Fig. 17 Spectrum of comet C/2007 N3 (Lulin) on 31 Jan 2009 with the sum of the telluric and best-fit synthetic fluorescent emission model (red) overplotted. The best fit water ortho (orange) and para (purple) models are shown. Also shown is $\mathrm{OH}$ prompt emission (olive). To illustrate the quality of the fit, the residual (scaled by a factor of two) with the best-fit models subtracted is shown. The 1-sigma error envelope is overplotted in green. The best fit is for statistical equilibrium $\left(T_{\text {spin }}>60 \mathrm{~K}\right)$

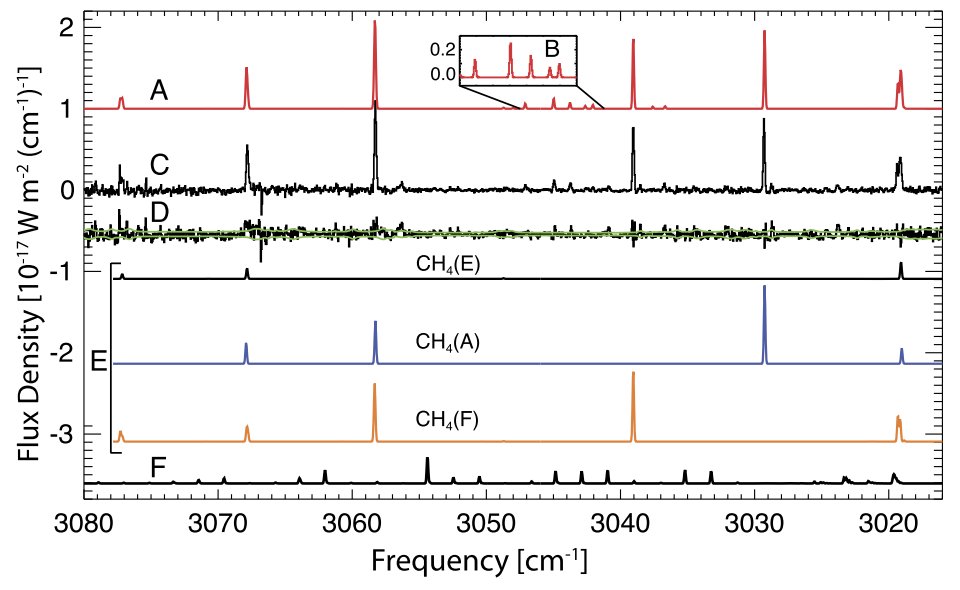

Fig. 18 Residual spectrum of comet C/2007 N3 (Lulin) on 31 Jan 2009 (C) with the sum of the best-fit synthetic fluorescent emission model (red) overplotted (A) and offset vertically for clarity. The OH prompt emission is shown in the inset (B). To illustrate the quality of the fit, the residual (scaled by a factor of two) with the best-fit models subtracted is shown in (D) with the 1-sigma error envelope overplotted in green. Beneath the residuals, individual fluorescent emission models are shown for the three spin components of $\mathrm{CH}_{4}(\mathbf{E})$ and $\mathrm{CH}_{3} \mathrm{D}(\mathbf{F})$ expanded vertically for clarity. The best fit is for statistical equilibrium $\left(T_{\text {spin }}>60 \mathrm{~K}\right)$. Adapted from Gibb et al. (2012)

The meaning of spin temperatures has been the subject of much debate. For an isolated molecule of water or methane in the gas phase, for example, nuclear spin conversion is strongly forbidden. For this reason, spin temperature has been considered a cosmogonic indicator in comets that can provide information on the formation temperature (Crovisier 1994; Mumma et al. 1987). Observational evidence supports the suggestion that OPRs do not change once volatiles are released into the gas phase. For example, recent results map- 


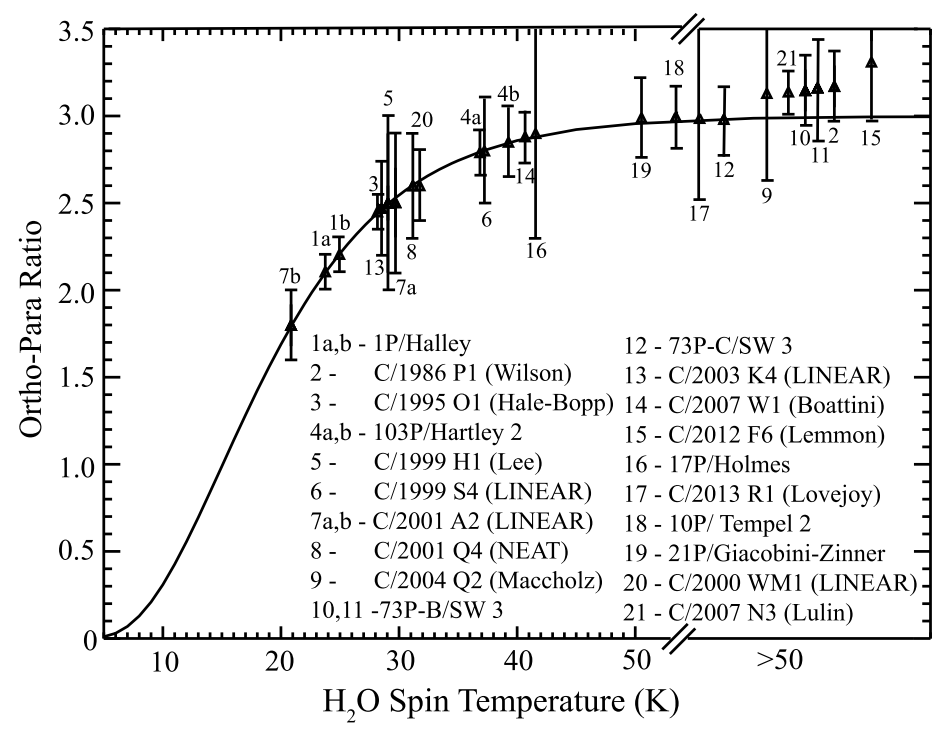

Fig. 19 OPR vs. Spin Temperature in water with measurements for individual comets. Individual OPR measurements are from Mumma et al. (1988: 1a, 1b, 2); Crovisier et al. (1997: 3, 1999: 4a, b); Dello Russo et al. (2005: 5, 6, 7a, 7b, 2007: 11, 12, 2008: 16); Kawakita et al. (2006: 8); Bonev et al. (2009: 9, 2008: 10); Woodward et al. (2007: 13); Villanueva et al. (2011: 14); Paganini et al. (2014: 15, 2015: 17, 2012: 18); DiSanti et al. (2013: 19); Radeva et al. (2010: 20); Gibb et al. (2012: 21)

ping the spin and rotational temperatures in cometary comae are consistent with a scenario in which the OPR is unchanged by coma processes (Bonev et al. 2008). Also, the OPR for $\mathrm{NH}_{2}$ does not correlate with the heliocentric distance (and hence rotational temperature) of the comet, nor does it correlate significantly with abundances of other volatiles (Shinnaka et al. 2011).

However, it has also been suggested that the spin temperature of water depends on the evaporation history of the ice (Buntkowsky et al. 2008). Hama et al. (2011) found experimentally that the OPR of water desorbed from ice after being vapor deposited at $8 \mathrm{~K}$ or formed via irradiation of a $\mathrm{CH}_{4} / \mathrm{O}_{2}$ mixture at $8 \mathrm{~K}$ was consistent with statistical equilibrium, suggesting that OPR may equilibrate during the thermal desorption process or that energetically produced water does not undergo nuclear spin conversion (to a lower OPR) in water ice. Both formation processes for water in the Hama et al. (2011) experiment were energetic, and the authors note that the spin temperature of water molecules produced by non-energetic processes must also be studied before definitive conclusions can be drawn, especially since cometary volatiles may have formed by low-energy processes. Nonetheless, such studies raise the possibility that cometary OPRs may not, in fact, be cosmogonic.

Can the measurements of OPR in comets shine any light on this question? We show the measurements of cometary OPR in water to date in Fig. 19. Water was chosen due to the greater number of OPR measurements than for other molecules in comets. Early measurements suggested a clustering near $T_{\text {spin }} \sim 30 \mathrm{~K}$, leading many authors to conclude a formation temperature of $\sim 30 \mathrm{~K}$ for a large population of comets. Similar conclusions have been drawn from studies based on $\mathrm{CH}_{4}$ (Kawakita et al. 2006) and $\mathrm{NH}_{2}$ (Shinnaka et al. 2011), particularly given the lack of correlation between spin temperature and other properties of the observed comets (Shinnaka et al. 2011). However, it must be noted that among the 20 comets measured to date, nearly all of them are within 1- or 2-sigma of 
the statistical equilibrium value, excepting the measurements reported for $1 \mathrm{P} / \mathrm{Halley}$, one of the two measurements for C/2001 A2, and C/1995 O1 (Hale-Bopp). Likewise, half of all measurements are at or above the statistical equilibrium value. It is also notable that no measurements exist below a spin temperature of $20 \mathrm{~K}$, and this observation holds for methane and ammonia spin temperatures as well.

Possible interpretations of spin temperatures measured in comets are therefore (1) that it is a cosmogonic indicator that gives the formation temperature of the molecule in question and therefore perhaps the formation conditions of the comet, (2) that it is a measurement that, while unchanged by coma processes, is set by processes occurring in the solid ice during or prior to sublimation. Current data are not sufficient to draw firm conclusions at this point. Even if further observations result in most comets consistent with statistical equilibrium, we may still be sampling cosmogonic material since it is known that comets contain material processed at high temperatures, such as crystalline silicates, which are interpreted to originate from turbulent radial mixing in the solar nebula (Bockelée-Morvan et al. 2002). Mixing of volatile material that formed at high temperatures in the inner disk to the comet forming region would have resulted in a higher OPR than for uncontaminated material that formed in the cold midplane. Clearly a great deal more work needs to be done to understand the significance of molecular spin states in cometary material.

\section{On the Origin of Neutral Sodium in Comet Tails}

The presence of sodium D line emission has been confirmed in a large number of comets close to perihelion since it was first reported more than a century ago (Bredichin 1882). Observations of comet C/1995O1 Hale-Bopp during the spring of 1997 led to the discovery of a new tail connected with the sodium $\mathrm{D}$ line emission. This neutral sodium gas tail is entirely different from the previously known ion and dust tails, and its associated source is unclear. Up to now, only physical reasons have been advanced to rationalize its origin. It has been proposed that this third type of tail is shaped by radiation pressure due to resonance fluorescence of sodium atoms (Cremonese et al. 1997). Further possibilities were considered and then rejected, as photo-sputtering and/or ion sputtering of nonvolatile dust grains (Ip and Jorda 1998). In the same way, collisions between the cometary dust and very small grains were also questioned (Ip and Jorda 1998). The scenario presented in this review is completely different since entirely based upon chemical grounds. It is shown that the $\mathrm{Na}^{+}$ ions, washed out of the refractory material during the hydration phase, loose their positive charge to evolve progressively into neutral species with their migration towards the surface of cometary ices.

Ellinger et al. (2015) follow the chemical path of sodium, starting from the incorporation into the ice until the final transformation into a neutral atom when released from the sublimating crystalline (see Sect. 2.5) cometary ice. The modeling of bulk, surface structures and reactivity of ices has been essentially developed for environmental applications (Casassa and Pisani 2002; Casassa et al. 2005; Calatayud et al. 2003) Levering on these results, high-level numerical simulations based on first principle periodic density functional theory (DFT) were used to describe the solid structure of the ice in the form of apolar hexagonal ice (Bussolin et al. 1998) composed of bi-layers of water molecules.

A code, specifically designed for the study of periodic systems, namely, the Vienna $a b$ initio simulation package (VASP) (Kresse and Hafner 1994a, 1994b) was used to carry out all the calculations. The generalized gradient approximation (GGA) was employed in the form of the Perdew and Wang exchange-correlation functional (Perdew et al. 1992) coupled 
Fig. 20 Typical structures of $\mathrm{Na}-\mathrm{H}_{2} \mathrm{O}_{\text {ice }}$ arrangements. Top: $\mathrm{Na}$ in the cavity inside hexagonal ice. Middle: $\mathrm{Na}$ replacing $\mathrm{H}_{2} \mathrm{O}$ in the crystal. Bottom: $\mathrm{Na}$ adsorbed on the surface of the ice. Color code: yellow $(\mathrm{Na})$; red $(\mathrm{O})$; white $(\mathrm{H})$
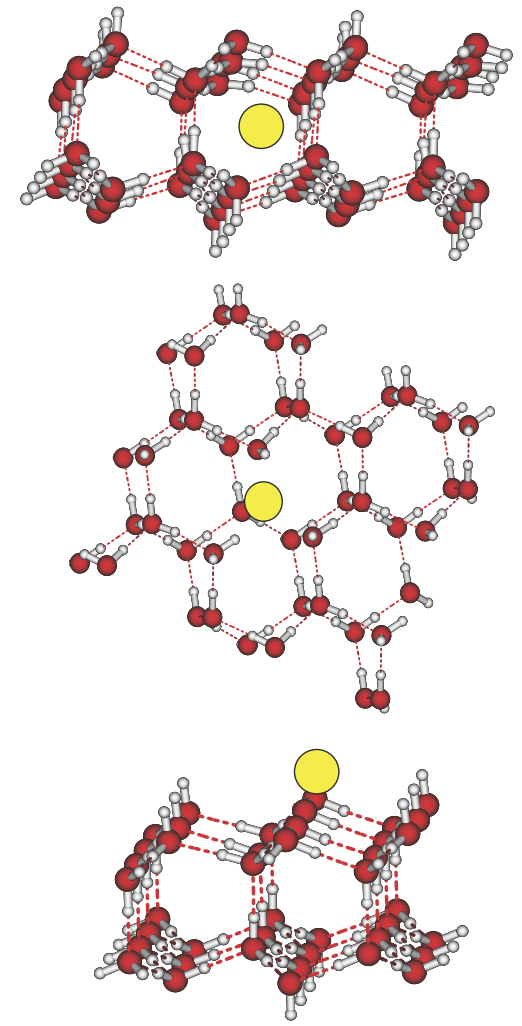

to the Grimme correction (Grimme et al. 2010) to take care of the long-range van der Waals interactions. The evolution of the ionic character of the sodium atom as it reaches the ice surface was obtained by a topological analysis (Silvi and Savin 1994) performed by means of the TOPMOD package (Llusar et al. 1999) and its recent extension to periodic systems (Kozlowski and Pilmé 2011).

A first calculation on isolated $\mathrm{Na}\left(\mathrm{H}_{2} \mathrm{O}\right)$ showed that the interaction of $\mathrm{Na}$ with one $\mathrm{H}_{2} \mathrm{O}$ is similar to that between two $\mathrm{H}_{2} \mathrm{O}$ molecules in the $\mathrm{H}_{2} \mathrm{O}$ dimer $(\approx 0.3 \mathrm{eV})$, which gives a hint in view of a possible replacement of a molecule of water by a sodium atom in organized structures. In liquid water, microsolvatation of sodium atoms has been studied on small clusters of $\mathrm{Na}\left(\mathrm{H}_{2} \mathrm{O}\right) n(n=1-6)$ to mimic the water environment. Both the structures in which $\mathrm{Na}$ is positioned on the surface and the encapsulated structure in which $\mathrm{Na}$ is surrounded by a solvation shell are minima on the potential surface. They are very close in energy (Hashimoto and Morokuma 1994).

In the ice, the periodic structure of the crystal imposes strong geometrical constraints. The most stable structure, not found in the liquid, is the one where $\mathrm{Na}$ takes the place of a water molecule in the lattice (middle structure in Fig. 20) when the ice is formed $(\Delta E \approx$ $1 \mathrm{eV})$. Contrary to microsolvatation, the stability of Na encapsulated in the bulk of the crystal $(\Delta E \approx 0.5 \mathrm{eV})$, shown in the top structure in Fig. 20) differs from that of the adsorbed $\mathrm{Na}$ according to the position on the surface. For example, the adsorption energies vary from ( $\Delta E \approx 0.6$ to $\approx 0.5$ and $\approx 0.1 \mathrm{eV}$ ) when the adsorption takes place over the center of a surface hexagon, over a surface oxygen (bottom structure in Fig. 20) and over an $\mathrm{OH}$ dangling bond, respectively. 
The most relevant point to this study is that the charge $q_{\mathrm{Na}}$ on the sodium atom depends strongly on the position with respect to the ice surface, as does the adsorption energy. When constrained to stay in the cavity inside the hexagonal lattice, Na remains largely ionized $\left(q_{\mathrm{Na}} \approx+0.9\right)$ which is close to the value of $\approx+0.8$ when Na takes the place of an $\mathrm{H}_{2} \mathrm{O}$ molecule in the bulk. When $\mathrm{Na}$ is adsorbed on top of an oxygen of the surface layer, $q_{\mathrm{Na}} \approx+0.2$, a value similar to that found for the isolated $\mathrm{Na}\left(\mathrm{H}_{2} \mathrm{O}\right)$ complex. Then, as the ice is progressively eroded by the radiation field, $\mathrm{Na}$ eventually reaches the surface where it can desorb as a neutral atom when the upper layer of the ice vaporizes.

\section{Summary}

The observed compositions of comets are the end product of a chemical history that began in the cold parent molecular cloud core. The change in physical conditions experienced by the core material as the core collapsed and the protosun and protosolar disk formed and evolved led to chemical changes that are reflected in the cometary volatiles that we observe today. Each stage of evolution left its imprint in the mixing ratios of molecules observed in comets and disentangling their effects requires interaction between comet and disk researchers. The combination of the two fields of study provide a powerful tool in understanding the drivers that determine comet composition and conversely what the variations in comet composition can tell us about the relative formation conditions of these comets.

Protostellar disks provide complementary information to the comet observations. Their chemistry is complex, covering ice chemistry in the cold, shielded midplane, warm gas phase chemistry and hot, highly ionized chemistry. Comets form from the ices in the midplane, yet dynamical processes ensure that this region does not evolve in isolation but rather is the end product of material that has been processed in a variety of different environments and then brought together in the cometary ices. Establishing the origin of cometary ices therefore requires not only studying the midplane but also an understanding the chemical and physical evolution of the disk as a whole. By studying disks we can observe the chemistry and physics that shaped the solar system as they shape other protoplanetary systems.

Molecular deuteration is a link that might tie together the disparate objects of the solar system and their formation history and give us the picture of the whole solar system formation history. However, at present this link has still weak connections that need to be reinforced. The advent of the powerful new facilities, as for example ALMA and IRAMNOEMA, will certainly allow this field to progress rapidly in the next few years.

Rosetta will provide detailed ground-truths for the organic and isotopic makeup of cometary volatiles. The new insights obtained into the chemical processes which likely produced these molecules-interstellar or nebular-will allow us to constrain models of the formation and evolution of the early solar system. Its instruments will characterize the composition of the comet 67P/Churyumov-Gerasimenko and distinguish between the molecules native to the comet nucleus (parent species) and those generated by chemistry or by fragmentation in the comae (daughter species). The observations it will make will provide new insights into the formation conditions of the comet. They will also elucidate the degree to which interstellar material can survive its journey into the solar nebula and from there into comets.

Several measurements will characterize the its thermal history, e.g., measurements of molecular spin temperatures (Gulkis and Alexander 2008) and deuteration and of the abundance of argon (Hassig et al. 2015). The relative abundances of elements can tell us something about the dominant molecular form of each element at the time of formation-if an 
element is depleted relative to others then the major carrier of that element must have been too volatile to be condensed at the time of formation, or must have desorbed in the intervening time. In particular Rosetta measurements may help to elucidate the mystery of the lack of nitrogen in comets (Altwegg et al. 2015).

A key measurement will be the ratio of oxygen isotopes in water. Meteoritic water has a very different ratio of the oxygen isotopes compared to oxygen isotopes in the Sun. Rosetta provides our best opportunity to directly detect the isotope ratio from the early solar system, and in so doing will distinguish between the various theoretical models of how the oxygen isotope fractionation occurred.

In summary, Rosetta's observations will provide new insights into the chemical processes that resulted in the formation of cometary volatiles and consequently into the conditions that were present in the solar nebula at their time of formation.

Acknowledgements The work of K. Willacy and C. Alexander was carried out at the Jet Propulsion Laboratory, California Institute of Technology, under a contract with the National Aeronautics and Space Administration. S.B. Charnley and S.N. Milam acknowledge the support of the Goddard Center for Astrobiology. Support for K. Willacy, S.B. Charnley and S.N. Milam was partially provided by the NASA Origins of Solar Systems Program. E. Gibb was supported by the NASA Exobiology and Evolutionary Biology (grant NNX11AG44G) and NSF Planetary Astronomy (grant AST-1211362) programs. O. Mousis and C. Ceccarelli acknowledge support from CNES. Support for O. Mousis was provided by the A*MIDEX project (no. ANR11-1DEX-0001-02) funded by the "Investissements d'Avenir" French government program managed by the French National Research Agency (ANR). M. Ali-Dib was supported by a grant from the city of Beançon. E.S. Wirström was supported by the Swedish National Space Board.

(C)2014 Jet Propulsion Laboratory, California Institute of Technology. All rights reserved.

\section{References}

F.C. Adams, The birth environment of the solar system. Annu. Rev. Astron. Astrophys. 48, 47 (2010)

M.F. A'Hearn, L.M. Feaga, H.U. Keller, H. Kawakita, D.L. Hampton, J. Kissel, K.P. Klaasen, L.A. McFadden, K.J. Meech, P.H. Schultz, J.M. Sunshine, P.C. Thomas, J. Veverka, D.K. Yeomans, S. Besse, D. Bodewits, T.L. Farnham, O. Groussin, M.S. Kelley, C.M. Lisse, F. Merlin, S. Protopapa, D.D. Wellnitz, Cometary volatiles and the origin of comets. Astrophys. J. 758, 29 (2012)

Y. Aikawa, Cold CO gas in protoplanetary disks. Astrophys. J. 656, L93 (2007)

Y. Aikawa, E. Herbst, Deuterium fractionation in protoplanetary disks. Astrophys. J. 526, 314 (1999)

Y. Aikawa, T. Nomura, Physical and chemical structure of protoplanetary disks with grain growth. Astrophys. J. 642, 1152 (2006)

Y. Aikawa, T. Umebayashi, T. Nakano, S.M. Miyama, Evolution of molecular abundances in protoplanetary disks with accretion flows. Astrophys. J. 519, 705 (1999)

Y. Aikawa, V. Wakelam, R.T. Garrod, E. Herbst, Molecular evolution and star formation: From prestellar cores to protostellar cores. Astrophys. J. 674, 984 (2008)

Y. Aikawa, V. Wakelam, F. Hersant, R.T. Garrod, E. Herbst, From prestellar to protostellar cores II. Time dependence and deuterium fractionation. Astrophys. J. 760, 40 (2012)

V. Akimkin, S. Zhukovska, D. Wiebe, D.A. Semenov, Ya. Pavlyuchenkov, A. Vasyunin, T. Birnstiel, Th. Henning, Protoplanetary disk structure with grain evolution: The ANDES model. Astrophys. J. 766, 8 (2013)

T. Albertsson, D.A. Semenov, Th. Henning, Modeling deuterium fractionation in cold and warm molecular environments with large chemical networks. Astrophys. J. Suppl. Ser. 207, 27 (2011)

T. Albertsson, D.A. Semenov, A.I. Vasyunin, Th. Henning, E. Herbst, New extended deuterium fractionation model: Assessment at dense ISM conditions and sensitivity analysis. Astrophys. J. Suppl. Ser. 207, 27 (2013)

T. Albertsson, D.A. Semenov, Th. Henning, Chemodynamical deuterium fractionation in the early solar nebula: The origins of water on Earth and in asteroids and comets. Astrophys. J. 784, 39 (2014)

M. Ali-Dib, O. Mousis, J.-M. Petit, J.I. Lunine, The measured compositions of Uranus and Neptune from their formation on the CO iceline. Astrophys. J. 793, 9 (2014)

Y. Alibert, O. Mousis, W. Benz, On the volatile enrichments and composition of Jupiter. Astrophys. J. 622, L145 (2005a) 
Y. Alibert, O. Mousis, C. Mordasini, W. Benz, New Jupiter and Saturn formation models meet observations. Astrophys. J. 626, L57 (2005b)

ALMA Partnership et al., First results from high angular resolution ALMA observations toward the HL Tau region. Astrophys. J. Lett. (2015, in press). arXiv:1503.02649

K. Altwegg, H. Balsiger, A. Bar-Nun, J.J. Berthelier, A. Bieler, P. Bochsler, C. Briois, U. Calmonte, M. Combi, J. De Keyser, P. Eberhardt, B. Fiethe, S. Fuselier, S. Gasc, T.I. Gombosi, K.C. Hansen, M. Hässig, E. Kopp, A. Korth, A. Jäckel, L. LeRoy, U. Mall, B. Marty, O. Mousis, E. Neefs, T. Owen, H. Rème, M. Rubin, T. Sémon, C. Tzou, H. Waite, P. Wurz, Comet 67P/Churyumov-Gerasimenko, a Jupiter family comet with a high D/H ratio. Science 347, 387 (2015)

M. Asplund, N. Grevesse, A.J. Sauval, P. Scott, The chemical composition of the Sun. Annu. Rev. Astron. Astrophys. 47, 481 (2009)

S.A. Balbus, J.F. Hawley, A powerful local shear instability in weakly magnetized disks. I-Linear analysis. II-Nonlinear evolution. Astrophys. J. 376, 214 (1991)

N. Biver, D. Bockelée-Morvan, J. Crovisier, D.C. Lis, R. Moreno, P. Colom, F. Henry, F. Herpin, G. Paubert, M. Womack, Radio wavelength molecular observations of comets C/1999 T1 (McNaught-Hartley), C/2001 A2 (LINEAR), C/2000 WM 1 (LINEAR) and 15P/Ikeya-Zhang. Astron. Astrophys. 449, 1255 (2006)

D. Bockelée-Morvan, D. Gautier, D.C. Lis, K. Young, J. Keene, T. Phillips, T. Owen, J. Crovisier, P.F. Goldsmith, E.A. Bergin, D. Depois, A. Wootten, Deuterated water in comet C/1996 B2 (Hyakutake) and its implications for the origin of comets. Icarus 133, 147 (1998)

D. Bockelée-Morvan, D. Gautier, F. Hersant, J.-M. Huré, F. Robert, Turbulent radial mixing in the solar nebula as the source of crystalline silicates in comets. Astron. Astrophys. 384, 1107 (2002)

D. Bockelée-Morvan, J. Crovisier, M.J. Mumma, H.A. Weaver, The composition of cometary volatiles, in Comets II (2004), p. 391

D. Bockelée-Morvan, N. Biver, B. Swinyard, M. de Val-Borro, J. Crovisier, P. Hartogh, D.C. Lis, R. Moreno, S. Szutowicz, E. Lellouch, M. Emprechtinger, G.A. Blake, R. Courtin, C. Jarchow, M. Kidger, M. Küppers, M. Rengel, G.R. Davis, T. Fulton, D. Naylor, S. Sidher, H. Walker, Herschel measurements of the $\mathrm{D} / \mathrm{H}$ and ${ }^{16} \mathrm{O} /{ }^{18} \mathrm{O}$ ratios in water in the Oort-cloud comet $\mathrm{C} / 2009 \mathrm{P} 1$ (Garradd). Astron. Astrophys. 544, 15 (2012)

D. Bockelée-Morvan, U. Calmonte, S. Charnley, J. Duprat, C. Engrand, A. Gicquel, M. Hässig, E. Jehin, H. Kawakita, B. Marty, S. Milam, A. Morse, P. Rousselot, S. Sheridan, E. Wirström, Cometary isotopic measurements. Space Sci. Rev. (2015, this issue). doi:10.1007/s11214-015-0156-9

B.P. Bonev, M.J. Mumma, H. Kawakita, H. Kobayashi, G.L. Villanueva, IRCS/Subaru observations of water in the inner coma of comet 73P-B/Schwassman-Wachmann 3: Spatially resolved rotational temperatures and ortho-para ratios. Icarus 196, 241 (2008)

B.P. Bonev, M.J. Mumma, E.L. Gibb, M.A. DiSanti, G.L. Villanueva, K. Magee-Sauer, R.S. Ellis, Comet C/2004 Q2 (Machholz): Parent volariles, a search for deuterated methane, and constraint on the $\mathrm{CH}_{4}$ spin temperature. Astrophys. J. 699, 1563 (2009)

B.P. Bonev, E. Gibb, G. Villanueva, M. DiSanti, L. Paganini, M. Mumma, J. Keane, K. Meech, K. Wright, E. Sudholt, M. Besic, $\mathrm{OH}^{*}$ prompt emission and near-infrared searches for HDO in the moderately bright comets C/2007 N3 Lulin and 103P/Hartley 2, in Asteroids, Comets and Meteors (2014)

T. Bredichin, Les vapeurs du sodium dans la comète de Wells. Astron. Nachr. 102, 207 (1882)

G. Buntkowsky, H.-H. Limbach, B. Walaszek, A. Adamczyk, Y. Xu, H. Breitzke, A. Schweitzer, T. Gutmann, M. Wachtler, J. Frydel, Th. Emmler, N. Amadeu, D. Tietze, B. Chaudret, Mechanisms of dipolar ortho/para $\mathrm{H}_{2} \mathrm{O}$ conversion in ice. Z. Phys. Chem. 222, 1049 (2008)

G. Bussolin, S. Casassa, P. Pisani, P. Ugliengo, Ab initio study of $\mathrm{HCl}$ and $\mathrm{HF}$ interaction with crystalline ice. I. Physical adsorption. J. Chem. Phys. 108, 9516 (1998)

M. Calatayud, D. Courmier, C. Minot, Ionization of $\mathrm{HCl}$ and $\mathrm{HF}$ in ice: a periodic DFT study. Chem. Phys. Lett. 369, 287 (2003)

J.S. Carr, J.R. Najita, Organic molecules and water in the planet formation region of young circumstellar disks. Science 319, 1504 (2008)

S. Casassa, C. Pisani, Interaction of $\mathrm{HOCl}$ with a chlorinated ice surface to produce molecular clorine: An ab-initio study. J. Chem. Phys. 116, 9856 (2002)

S. Casassa, M. Calatayud, K. Doll, K.C. Minot, C. Pisani, Proton ordered cubic and hexagonal periodic models of ordinary ice. Chem. Phys. Lett. 409, 110 (2005)

P. Caselli, E. Keto, E.A. Bergin, M. Tafalla, Y. Aikawa, T. Douglas, L. Pagani, U.A. Yildiz, F.F.S. van der Tak, C.M. Walmsley, C. Codella, B. Nisini, L.E. Kristensen, E.F. van Dishoeck, First detection of water vapor in a pre-stellar core. Astrophys. J. Lett. L37, 5 (2012)

C. Ceccarelli, C. Dominik, B. Lefloch, P. Caselli, E. Caux, Detection of $\mathrm{H}_{2} \mathrm{D}^{+}$: Measuring the midplane degree of ionization in the disks of DM Tauri and TW Hydrae. Astrophys. J. 607, L51 (2004) 
C. Ceccarelli, P. Caselli, D. Bockelee-Morvan, O. Mousis, S. Pizzarello, F. Robert, D. Semenov, Deuterium fractionation: the Ariadne's thread from the pre-collapse phase to meteorites and comets today, in Protostars and Planets VI, ed. by H. Beuther, R.S. Klessen, C.P. Dullemond, Th. Henning (University of Arizona Press, Tucson, 2014). arXiv:1403.7143

S.B. Charnley, On the nature of interstellar organic chemistry, in Astronomical and biochemical origins and the search for life in the Universe, vol. 89, ed. by C. Batalli Cosmovici, S. Bowyer, D. Werthimer (Editrice Compositori, Bologna, 1997)

S.B. Charnley, S.D. Rodgers, Interstellar reservoirs of cometary matter. Space Sci. Rev. 138, 59 (2008)

S.B. Charnley, S.D. Rodgers, Clouds, clumps, cores and comets-a cosmic chemical connection? Adv. Geosci. 15, 211 (2009)

S.B. Charnley, A.G.G.M. Tielens, S.D. Rodgers, Deuterated methanol in the Orion Compact Ridge. Astrophys. J. 482, L203 (1997)

S.B. Charnley, P. Ehrenfreund, T.J. Millar, A.C.A. Boogert, A.J. Markwick, H.M. Butner, R. Ruiterkamp, S.D. Rodgers, Observational tests for grain chemistry: Posterior isotopic labeling. Mon. Not. R. Astron. Soc. 347, 157 (2004)

K.M. Chick, P. Cassen, Thermal processing of interstellar dust grains in the primitive solar environment. Astrophys. J. 477, 398 (1997)

F.J. Ciesla, The phases of water ice in the solar nebula. Astrophys. J. 784, L1 (2014)

F.J. Ciesla, S.B. Charnley, The physics and chemistry of nebular evolution, in Meteorites and the Early Solar System II, ed. by D.S. Lauretta, H.Y. McSween Jr. (University of Arizona Press, Tucson, 2006), p. 209

R.N. Clayton, Oxygen isotopes in meteorites. Annu. Rev. Earth Planet. Sci. 21, 115 (1993)

R.N. Clayton, L. Grossman, T.K. Mayeda, A component of primitive nuclear composition in carbonaceous meteorites. Science 182, 485 (1973)

L.I. Cleeves, F.C. Adams, E.A. Bergin, Exclusion of cosmic rays in protoplanetary disks: stellar and magnetic effects. Astrophys. J. 772, 5 (2013)

L.I. Cleeves, E.A. Bergin, C.M.O. 'D Alexander, F. Du, D. Graninger, K.I. Öberg, T.J. Harries, The ancient heritage of water ice in the solar system. Science 345, 1590 (2014a)

L.I. Cleeves, E.A. Bergin, F.C. Adams, Exclusion of cosmic rays in protoplanetary disks. II. Chemical gradients and observational signatures. Astrophys. J. 794, 123 (2014b)

A.L. Cochran, W.D. Cochran, E.S. Barker, $\mathrm{N}_{2}^{+}$and $\mathrm{CO}^{+}$in Comets 122P/1995 S1 (deVico) and C/1995 O1 (Hale-Bopp). Icarus 146, 583 (2000)

G. Cremonese, H. Boehnhardt, J. Crovisier, H. Rauer, A. Fitzsimmons, M. Fulle, J. Licandro, D. Pollacco, G.P. Tozzi, R.M. West, Neutral sodium from comet Hale-Bopp: A third type of tail. Astrophys. J. 490, L199 (1997)

J. Crovisier, The water molecule in comets-fluorescence mechanisms and thermodynamics of the inner coma. Astron. Astrophys. 130, 361 (1994)

J. Crovisier, The molecular composition of comets and its interrelation with other small bodies of the Solar System, in IAU Symp 229 “Asteroids, Comets, Meteors”, ed. by L. Daniela, M. Sylvio Ferraz, F.J. Angel (2006), p. 113

J. Crovisier, K. Leech, D. Bockelée-Morvan, T.Y. Brooke, M.S. Hanner, B. Altieri, H.U. Keller, E. Lellouch, The spectrum of comet Hale-Bopp (C/1995 O1) observed with the Infrared Space Observatory at 2.9 AU from the Sun. Science 275, 1904 (1997)

J. Crovisier, Th. Encrenaz, E. Lellouch, E. Bockelée-Morvan, B. Altieri, K. Leech, A. Salama, M.J. Griffin, Th. de Graauw, E.F. van Dishoeck, R. Knacke, T.Y. Brooke, ISO spectroscopic observations of short-period comets, in The Universe as Seen by ISO, ed. by P. Cox, M.F. Kessler. ESA SP-427 (ESA, Noordwijk, 1999), p. 161

J. Crovisier, D. Bockelée-Morvan, P. Colom, N. Biver, D. Despois, D.C. Lis, The composition of ices in comet C/1995 O1 (Hale-Bopp) from radio spectroscopy: Further results and upper limits on undetected species. Astron. Astrophys. 418, 1141 (2004)

P. D’Alessio, B. Merin, N. Calvet, L. Hartmann, B. Montesinos, WWW database of models of accretion disks irradiated by the central star. Rev. Mex. Astron. Astrofís. 41, 61 (2005)

I. de Gregorio-Monsalvo et al., Unveiling the gas-and dust disk structure in HD 16329 using ALMA observations. Astron. Astrophys. 557, 133 (2013)

I. de Pater, M. Richmond, Neptune's microwave spectrum from $1 \mathrm{~mm}$ to $20 \mathrm{~cm}$. Icarus 80, 1 (1989)

I. de Pater, P.N. Romani, S.K. Atreya, Uranus deep atmosphere revealed. Icarus 82, 288 (1989)

N. Dello Russo, B.P. Bonev, M.A. DiSanti, M.J. Mumma, E.L. Gibb, K. Magee-Sauer, R.J. Barber, J. Tennyson, Water production rates, rotational temperatures, and spin temperatures in comets C/1999 H1 (Lee), C/1999 S4, and C/2001 A2. Astrophys. J. 621, 537 (2005)

N. Dello Russo, R.J. Vervack, H.A. Weaver, N. Biver, D. Bockelée-Morvan, J. Crovisier, C.M. Lisse, Compositional homogeneity in the fragmented comet 73P/Schwassmann-Wachmann 3. Nature 448, 172 (2007) 
N. Dello Russo, R.J. Vervack, H.A. Weaver, M.M. Montgomery, R. Deshpande, Y.R. Fernández, E.L. Martin, The volatile composition of comet 17P/Holmes after its extraordinary outburst. Astrophys. J. 680, 793 (2008)

M.A. DiSanti, B.P. Bonev, G.L. Villanueva, M.J. Mumma, Highly depleted ethane and mildly depleted methanol in comet 21P/Giacobini-Zinner: Application of a new empirical $v_{2}$-band model for $\mathrm{CH}_{3} \mathrm{OH}$ near 50 K. Astrophys. J. 763, 1 (2013)

S.E. Dodson-Robinson, K. Willacy, P. Bodenheimer, N.J. Turner, C.A. Beichman, Ice lines, planetesimal composition and solid surface density in the solar nebula. Icarus 200, 672 (2009)

A.Z. Dolginov, T.F. Stepinski, Are cosmic rays effective for ionization of protoplanetary disks? Astrophys. J. 427, 377 (1994)

C. Dominik, C. Ceccarelli, D. Hollenbach, M. Kaufman, Gas-phase water in the surface layers of protoplanetary disks. Astrophys. J. 635, 85 (2005)

C.P. Dullemond, C. Dominik, The effect of dust settling on the appearance of protoplanetary disks. Astron. Astrophys. 421, 1075 (2004)

A. Dutrey, D. Semenov, E. Chapillon, U. Gorti, S. Guilloteau, F. Hersant, M. Hogerheijde, M. Hughes, G. Meeus, H. Nomura, V. Piétu, C. Qi, V. Wakelam, Physical and chemical structure of planet-forming disks probed by millimeter observations and modeling, in Protostars and Planets VI, ed. by H. Beuther, R. Klessen, C. Dullemond, Th. Henning (University of Arizona Press, Tuscon, 2014), p. 317

P. Eberhardt, D. Krankowsky, W. Schutte, U. Dolder, P. Limmerzahl, J.J. Berthelier, J. Woweries, U. Stubbemann, R.R. Hodges, J.H. Hoffman, J.M. Illiano, The $\mathrm{CO}$ and $\mathrm{N}_{2}$ abundance in comet P/Halley. Astron. Astrophys. 187, 481 (1987)

P. Eberhardt, M. Reber, D. Krankowsky, R.R. Hodges, The D/H and ${ }^{18} \mathrm{O} /{ }^{16} \mathrm{O}$ ratios in water from comet P/Halley. Astron. Astrophys. 302, 301 (1995)

Y. Ellinger, F. Pauzat, O. Mousis, A. Guilbert-Lepoutre, F. Leblanc, M. Ali-Dib, M. Doronin, E. Zicler, A. Doressoundiram, Neutral sodium in cometary tails as a remnant of early aqueous alteration. Astrophys. J. Lett. 801, L30 (2015)

G.J. Flynn, Physical, chemical, and mineralogical properties of comet 81P/Wild 2 particles collected by Stardust. Earth Moon Planets 102, 447 (2008)

J.K.J. Fogel, T.J. Bethell, E.A. Bergin, N. Calvet, D. Semenov, Chemistry of a protoplanetary disk with grain settling and Ly $\alpha$ radiation. Astrophys. J. 726, 29 (2011)

K. France, E. Schindhelm, E.A. Bergin, E. Roueff, H. Abgrall, High-resolution ultraviolet radiation fields of classical T Tauri stars. Astrophys. J. 127, 20 (2014)

N. Fray, B. Schmitt, Sublimation of ices of astrophysical interest: A bibliographic review. Planet. Space Sci. 57, 2053 (2009)

K.Y. Furuya, Y. Aikawa, H. Nomura, F. Hersant, V. Wakelam, Water in protoplanetary disks: Deuteration and turbulent mixing. Astrophys. J. 779, 11 (2013)

D. Gautier, F. Hersant, O. Mousis, J.I. Lunine, Enrichments in volatiles in Jupiter: A new interpretation of the Galileo measurements. Astrophys. J. 550, L227 (2001)

E.L. Gibb, B.P. Bonev, G. Villanueva, M.A. DiSanti, M.J. Mumma, E. Sudholt, Y. Radeva, Chemical composition of comet C/2007 N3 (Lulin): Another "atypical" comet. Astrophys. J. 750, 102 (2012)

A.E. Glassgold, J. Najita, J. Igea, X-ray ionization of proto-planetary disks. Astrophys. J. 480, 344 (1997)

S. Grimme, J. Antony, S. Ehrlich, H. Krieg, A consistent and accurate ab initio parameterization of density functional dispersion correction (DFT-D) for the 94 elements H-Pu. J. Chem. Phys. 132, 154104 (2010)

S. Gulkis, C. Alexander, Composition measurements of a comet from the Rosetta Orbiter spacecraft. Space Sci. Rev. 138, 259 (2008)

T. Hama, N. Watanabe, A. Kouchi, M. Yokoyama, Spin temperature of water molecules desorbed from the surfaces of amorphous solid water, vapor-deposited and produced from photolysis of a $\mathrm{CH}_{4} / \mathrm{O}_{2}$ solid mixture. Astrophys. J. Lett. 738, 15 (2011)

D. Harsono, R. Visser, S. Bruderer, E.F. van Dishoeck, L.E. Kristensen, Evolution of CO lines in timedependent models of protostellar disk formation. Astron. Astrophys. 555, 45 (2013)

P. Hartogh, D.C. Lis, D. Bockelée-Morvan, M. de Val-Borro, N. Biver, M. Küppers, M. Emprechtinger, E.A. Bergin, J. Crovisier, M. Rengel, R. Moreno, S. Szutowicz, G.A. Blake, Ocean-like water in the Jupiterfamily comet 103P/Hartley 2. Nature 478, 218 (2011)

K. Hashimoto, K. Morokuma, Ab-initio molecular-orbital study of $\mathrm{Na}\left(\mathrm{H}_{2} \mathrm{O}\right) \mathrm{N}(\mathrm{N}=1-6)$ clusters and their ions-comparison of electronic-structure of the surface and their interior complexes. J. Am. Chem. Soc. 116, 11436 (1994)

M. Hassig, K. Altwegg, J. Berthelier, U. Almonte, T. Gombosi, J. De Jeyser, B. Fiethe, S. Fuselier, L. LeRoy, M. Rubin, The capabilities of ROSINA/DFMS to measure argon isotopes at comet 67P/ChuryumovGerasimenko. Planet. Space Sci. 105, 175 (2015)

J.F. Hawley, S.A. Balbus, A powerful local shear instability in weakly magnetized disks. Astrophys. J. 376, 223 (1991) 
J.F. Hawley, C.F. Gammie, S.A. Balbus, Local three-dimensional simulations of an accretion disk hydromagnetic dynamo. Astrophys. J. 464, 690 (1996)

A.N. Heays, R. Visser, R. Gredel, W. Ubachs, B.R. Lewis, S.T. Givson, E.F. van Dishoeck, Isotope selective photodissociation of $\mathrm{N}_{2}$ by the interstellar radiation field and cosmic rays. Astron. Astrophys. 562, 61 (2014)

J.E. Heidenreich III, M.H. Thiemens, A non-mass-independent isotope effect in the production of ozone from molecular oxygen. J. Chem. Phys. 78, 892 (1983)

D. Heinzeller, H. Nomura, C. Walsh, T.J. Millar, Chemical evolution of protoplanetary disks-the effects of viscous accretion, turbulent mixing and disk winds. Astrophys. J. 731, 115 (2011)

Th. Henning, D. Semenov, Chemistry in protoplanetary disks. Chem. Rev. 113, 9016 (2013)

E. Herbst, E.F. van Dishoeck, Complex organic interstellar molecules. Annu. Rev. Astron. Astrophys. 47, 427 (2009)

F. Hersant, V. Wakelam, A. Dutrey, S. Guilloteau, E. Herbst, Cold CO in circumstellar disks. On the effects of photodesorption and vertical mixing. Astron. Astrophys. 493, L49 (2009)

M.R. Hogerheijde, E.A. Bergin, C. Brinch, L.I. Cleeves, J.K. Fogel, G.A. Blacke, C. Dominik, D.C. Lis, G. Melnick, D. e Neufeld, O. Panić, J.C. Pearson, L. Kristensen, U.A. Yildiz, E.A. Dishoeck, Detection of the water reservoir in a forming planetary system. Science 354, 338 (2011)

R. Hueso, T. Guillot, Evolution of protoplanetary disks: Constraints from DM Tauri and GM Aurigae. Astron. Astrophys. 442, 703 (2005)

D. Hutsemékers, J. Manfroid, E. Jehin, J.M. Zucconi, C. Arpigny, The ${ }^{16} \mathrm{OH} /{ }^{18} \mathrm{OH}$ and OD/OH isotope ratios in comet C/2002 T7 (LINEAR). Astron. Astrophys. 490, 31 (2008)

M. Ilgner, R.P. Nelson, On the ionization fraction in protoplanetary disks. II. The effect of turbulence mixing on gas-phase chemistry. Astron. Astrophys. 445, 223 (2006)

M. Ilgner, R.P. Nelson, Turbulent transport and its effect on the dead zone in protoplanetary discs. Astron. Astrophys. 483, 815 (2008)

M. Ilgner, Th. Henning, A.J. Markwick, T.J. Millar, Transport processes and chemical evolution in steady accretion disk flows. Astron. Astrophys. 415, 643 (2004)

S. Inutsuka, T. Sano, Self-sustained ionization and vanishing dead zones in protoplanetary disks. Astrophys. J. 628, L155 (2005)

W.-H. Ip, L. Jorda, Can the sodium tail of comet Hale-Bopp have a dust-impact origin? Astrophys. J. 496, L47 (1998)

N. Iro, D. Gautier, F. Hersant, D. Bockelée-Morvan, J.I. Lunine, An interpretation of the nitrogen deficiency in comets. Icarus 161, 511 (2003)

W.M. Irvine, D. Bockelée-Morvan, D.C. Lis, H.E. Matthews, N. Biver, J. Crovisier, J.K. Davies, W.R.F. Dent, D. Gautier, P.D. Godfrey, J. Keene, A.J. Lovell, T.C. Owen, T.G. Phillips, H. Tauer, F.P. Schloerb, M. Senay, K. Young, Spectroscopic evidence for interstellar ices in comet Hyakutake. Nature 383, 418 (1996)

E. Jehin, J. Manfroid, D. Hutsemékers, C. Arpigny, J.-M. Zucconi, Isotopic ratios in comets: Status and perspectives. Earth Moon Planets 105, 167 (2009)

E.K. Jessberger, J. Kissel, Chemical properties of cometary dust and a note on carbon isotopes, in IAU Colloq. 116: Comets in the Post-Halley Era, vol. 167 (1991), p. 1075

J.J. Kavelaars, O. Mousis, J.-M. Petit, H.A. Weaver, On the formation location of Uranus and Neptune as constrained by dynamical and chemical models of comets. Astrophys. J. Lett. 734, L30 (2011)

H. Kawakita, H. Kobayashi, Formation conditions of icy materials in comet C/2004 Q2 (Machholz). II. Diagnostics using nuclear spin temperatures and deuterium-to-hydrogen ratios in cometary molecules. Astrophys. J. 693, 388 (2009)

H. Kawakita, J.-i. Watanabe, R. Furusho, T. Fuse, M.T. Capria, M.C. De Sanctis, G. Cremonese, Spin temperatures of ammonia and water molecules in comets. Astrophys. J. 601, 1152 (2004)

H. Kawakita, J.-I. Watanabe, R. Furusho, T. Fuse, D.C. Boice, Nuclear spin temperature and deuterium-tohydrogen ratio of methane in comet C/2001 Q4 (NEAT). Astrophys. J. Lett. 623, 49 (2005)

H. Kawakita, N. Dello Russo, R. Furusho, T. Fuse, J.-i. Watanabe, D.C. Boice, K. Sadakane, N. Arimoto, M. Ohkubo, T. Ohnishi, Ortho-to-para ratios of water and ammonia in comet C/2001 Q4 (NEAT): Comparison of nuclear spin temperatures of water and methane. Astrophys. J. 643, 1337 (2006)

A. Kouchi, T. Yamamoto, T. Kozasa, T. Kuroda, J.M. Greenberg, Conditions for condensation and preservation of amorphous ice and crystallinity of astrophysical ices. Astron. Astrophys. 290, 1009 (1994)

D. Kozlowski, J. Pilmé, J. Comput. Chem. 32, 3207 (2011)

G. Kresse, J. Hafner, Ab-initio molecular-dynamics simulation of the liquid-metal amorphous-semiconductor transition in germanium. Phys. Rev. B 49, 14251 (1994a)

G. Kresse, J. Hafner, Norm-conserving and ultrasoft pseudopotentials for first-row and transition elements. J. Phys. Condens. Matter 6, 8245 (1994b) 
W.D. Langer, T.E. Graedel, M.A. Frerking, P.B. Armentrout, Carbon and oxygen isotope fractionation in dense interstellar clouds. Astrophys. J. 27, 581 (1984)

T. Lee, A local proton irradiation model for isotopic anomalies in the solar system. Astrophys. J. 224, 217 (1978)

J.-E. Lee, E.A. Bergin, J.R. Lyons, Oxygen isotope anomalies of the Sun and the original environment of the solar system. Meteorit. Planet. Sci. 43, 1351 (2008)

E. Lellouch, C. de Bergh, B. Sicardy, H.U. Käufl, A. Smette, High resolution spectroscopy of Pluto's atmosphere: Detection of the $2.3 \mu \mathrm{m} \mathrm{CH}_{4}$ bands and evidence for carbon monoxide. Astron. Astrophys. 530, L4 (2011)

J.S. Lewis, R.G. Prinn, Kinetic inhibition of $\mathrm{CO}$ and $\mathrm{N}_{2}$ reduction in the solar nebula. Astrophys. J. 238, 357 (1980)

D.C. Lis, N. Biver, D. Bockelée-Morvan, P. Hartogh, E.A. Bergin, G.A. Blake, J. Crovisier, M. de Val-Borro, E. Jehin, M. Küppers, J. Manfroid, R. Moreno, M. Rengel, S. Szutowicz, A Herschel study of D/H in water in the Jupiter-family comet 45P/Honda-Mrkos-Pajdusáková and prospects for D/H measurements with CCAT. Astrophys. J. 774, L3 (2013)

R. Llusar, A. Beltran, J. Andrès, B. Silvi, Topological analysis of electron density in depleted homopolar chemical bonds. J. Comput. Chem. 20, 1517 (1999)

J.I. Lunine, D.J. Stevenson, Thermodynamics of clathrate hydrate at low and high pressures with application to the outer solar system. Astrophys. J. Suppl. Ser. 58, 493 (1985)

J.R. Lyons, E.D. Young, CO self-shielding as the origin of oxygen isotope anomalies in the early solar nebula. Nature 435, 193 (2005)

U. Marboeuf, O. Mousis, J.-M. Petit, B. Schmitt, Clathrate hydrates formation in short-period comets. Astrophys. J. 708, 812 (2010a)

U. Marboeuf, O. Mousis, J.-M. Petit, B. Schmitt, A.L. Cochran, H.A. Weaver, On the stability of clathrate hydrates in comets 67P/Churyumov-Gerasimenko and 46P/Wirtanen. Astron. Astrophys. 525, 144 (2010b)

U. Marboeuf, B. Schmitt, J.-M. Petit, O. Mousis, N. Fray, A cometary nucleus model taking into account all phase changes of water ice: Amorphous, crystalline, and clathrate. Astron. Astrophys. 542, 82 (2012)

G.S. Mathews, P.D. Klassen, A. Juhász, D. Harsono, E. Chapillon, E.F. van Dishoeck, D. Espada, I. de Gregorio-Monsalvo, A. Hales, M.R. Hogerheijde, J.C. Mottram, M.G. Rawlings, S. Takahasi, L. Testi, ALMA imaging of the CO snowline of the HD 163296 disk with DCO ${ }^{+}$. Astron. Astrophys. 557, 132 (2013)

J.S. Mathis, W. Rumpl, K.H. Nordsieck, The size distribution of interstellar grains. Astrophys. J. 217, 425 (1977)

K.D. McKeegan, A.P.A. Kallio, V.S. Heber, G. Jarzebinski, P.H. Mao, C.D. Coath, T. Kunihiro, R.C. Wiens, J.E. Nordholt, R.W. Moses, D.B. Reisenfeld, A.J.G. Jurewicz, D.S. Burnett, The oxygen isotopic composition of the Sun inferred from captured solar wind. Science 332, 1528 (2011)

R. Meier, T.C. Owen, Cometary deuterium. Space Sci. Rev. 1, 99 (1999)

R. Meier, T.C. Owen, D.C. Jewitt, H.E. Matthews, M. Senay, M.N. Biver, D. Bockelée-Morvan, J. Crovisier, D. Gautier, Deuterium in comet C/1995 O1 (Hale-Bopp): Detection of DCN. Science 279, 1707 (1998)

T.J. Millar, A. Bennett, E. Herbst, Deuterium fractionation in dense interstellar clouds. Astrophys. J. 340,906 (1989)

J.C. Mottram, E.F. van Dishoeck, M. Schmalzl, L.E. Kristensen, R. Visser, M.R. Hogerheijde, S. Bruderer, Waterfalls around protostars. Infall motions toward Class 0/1 envelopes as probed by water. Astron. Astrophys. 558, 126 (2013)

O. Mousis, Modeling the thermodynamical conditions in the Uranian subnebula-implications for regular satellite composition. Astron. Astrophys. 413, 373 (2004)

O. Mousis, D. Gautier, Constraints on the presence of volatiles in Ganymede and Callisto from an evolutionary turbulent model of the Jovian subnebula. Planet. Space Sci. 52, 361 (2004)

O. Mousis, D. Gautier, D. Bockelée-Morvan, F. Robert, B. Dubrulle, A. Drouart, Constraints on the formation of comets from D/H ratios measured in $\mathrm{H}_{2} \mathrm{O}$ and $\mathrm{HCN}$. Icarus 148, 513 (2000)

O. Mousis, D. Gautier, D. Bockelée-Morvan, An evolutionary turbulent model of Saturn's subnebula: Implications for the origin of the atmosphere of Titan. Icarus 156, 162 (2002)

O. Mousis, J.I. Lunine, C. Thomas, M. Pasek, U. Varboeuf, Y. Alibert, V. Ballenegger, D. Cordier, Y. Ellinger, F. Pauzat, S. Picaud, Clathration of volatiles in the Solar Nebula and implications for the origin of Titan's atmosphere. Astrophys. J. 691, 1780 (2009a)

O. Mousis, U. Marboeuf, J.L. Lunine, Y. Alibert, L.N. Fletcher, G.S. Orton, F. Pauzat, Y. Ellinger, Determination of the minimum masses of heavy elements in the envelopes of Jupiter and Saturn. Astrophys. J. 696, $1348(2009 b)$

O. Mousis, J.I. Lunine, S. Picaud, D. Cordier, Volatile inventories in clathrate hydrates formed in the primordial nebula. Faraday Discuss. 147, 509 (2010) 
O. Mousis, J.I. Lunine, N. Madhusudhan, T.V. Johnson, Nebular water depletion as the cause of Jupiter's low oxygen abundance. Astrophys. J. 751, L7 (2012a)

O. Mousis, A. Guilbert-Lepoutre, J.I. Lunine, A.L. Cochran, J.H. Waite, J.M. Petit, P. Rousselot, The dual origin of the nitrogen deficiency in comets: Selective volatile trapping in the nebula and postaccretion radiogenic heating. Astrophys. J. 757, 146 (2012b)

O. Mousis et al., Scientific rationale of Saturn's in situ exploration. Planet. Space Sci. 104, 29 (2015)

M.J. Mumma, S.B. Charnley, The chemical composition of comets-emerging taxonomies and natal heritage. Annu. Rev. Astron. Astrophys. 49, 471 (2011)

M.J. Mumma, H.A. Weaver, H.P. Larson, The ortho-para ratio of water vapor in comet P/Halley. Astron. Astrophys. 187, 419 (1987)

M.J. Mumma, W.E. Blass, H.A. Weaver, H.P. Larson, in The Formation and Evolution of Planetary Systems, Baltimore, ed. by H.A. Weaver, F. Paresce, L. Danly (Cambridge University Press, Cambridge, 1988), p. 157

K. Nagashima, A.N. Krot, H. Yurimoto, Stardust silicates from primitive meteorites. Nature 428, 921 (2004)

J.R. Najita, J.S. Carr, K.M. Pontoppidan, C. Salyk, E.F. van Dishoeck, G.A. Blake, The HCN-water ratio in the planet formation region of disks. Astrophys. J. 766, 134 (2013)

L.R. Nittler, Presolar stardust in meteorites: recent advances and scientific frontiers. Earth Planet. Sci. Lett. 209, 259 (2003)

G. Notesco, A. Bar-Nun, $\mathrm{A} \sim 25 \mathrm{~K}$ temperature of formation for the submicron ice grains which formed comets. Icarus 175, $546(2005)$

K.I. Öberg, R. Murray-Clay, E.A. Bergin, The effects of snowlines on C/O in planetary atmospheres. Astrophys. J. 743, L16 (2011)

T. Owen, P. Mahaffy, H.B. Niemann, S. Atreya, T. Donahue, A. Bar-Nun, I. de Pater, A low-temperature origin for the planetesimals that formed Jupiter. Nature 402, 269 (1999)

L. Pagani, E. Roueff, P. Lesaffre, Ortho- $\mathrm{H}_{2}$ and the age of interstellar dark clouds. Astrophys. J. 739, L35 (2011)

L. Paganini, M.A. DiSanti, M.J. Mumma, G.L. Villanueva, B.P. Bonev, J.V. Keane, E.L. Gibb, H. Boehnhard, K.J. Meech, The unexpectedly bright comet C/2012 F6 (Lemmon) unveiled at near-infrared wavelengths. Astron. J. 147, 15 (2014)

J.P. Perdew, J.I. Chevary, S.H. Vosko, K.A. Jackson, M.R. Pederson, D.J. Singh, C. Fiolhais, Atoms, molecules, solids and surfaces-applications of the generalized gradient approximation for exchange and correlation. Phys. Rev. B 46, 6671 (1992)

M.V. Persson, J.K. Jorgensen, E.F. van Dishoeck, D. Harsono, The deuterium fractionation of water on solar system scales in deeply-embedded low-mass protostars. Astron. Astrophys. 563, 74 (2014)

M. Pettini, R. Cooke, A new precise measurement of the primordial abundance of deuterium. Mon. Not. R. Astron. Soc. 425, 2477 (2012)

L. Podio, I. Kamp, C. Codella, S. Cabrit, B. Nisini, C. Dougados, G. Sandell, J.P. Williams, L. Testi, W.-F. Thi, P. Woitke, R. Meirjink, M. Spaans, G. Aresu, F. Ménard, C. Pinte, Water vapor in the protoplanetary disk of DG Tau. Astrophys. J. Lett. 766, L5 (2013)

K.M. Pontoppidan, S.M. Blevins, The chemistry of planet forming regions is not interstellar. Faraday Discuss. 168, 49 (2014)

K.M. Pontoppidan, C.P. Dullemond, E.F. van Dishoeck, G.A. Blake, A.C.A. Boogert, N.J. Evans II, J.E. Kessler-Silacii, F. Lahuis, Ices in the edge-on disk CRBR 2422.8-3423: Spitzer spectroscopy and Monte Carlo radiative transfer modeling. Astrophys. J. 622, 463 (2005)

K.M. Pontoppidan, C. Salyk, G.A. Blake, H.A. Käufl, Spectrally resolved pure rotational lines of water in protoplanetary disks. Astrophys. J. 722, L173 (2010)

K.M. Pontoppidan, C. Salyk, E.A. Bergin, S. Brittain, B. Marty, O. Mousis, K.I. Öberg, Volatiles in protoplanetary disks, in Protostars and Planets VI, ed. by H. Beuther, R. Klessen, C. Dullemond, Th. Henning (University of Arizona Press, Tucson, 2014), p. 363

C. Qi, D.J. Wilner, Y. Aikawa, G.A. Blake, M.R. Hogerheijde, Resolving the chemistry in the disk of TW Hydrae. I. Deuterated species. Astrophys. J. 681, 1396 (2008)

C. Qi et al., Resolving the CO snow line in the disk around HD 163296. Astrophys. J. 740, 84 (2011)

C. Qi, K.I. Öberg, D.J. Wilner, P. D’Alessio, E.A. Bergin, S.M. Andrews, G.A. Blake, M.R. Hogerheijde, E.F. van Dishoeck, Imaging of the CO snow line in a Solar Nebula analog. Science 341, 630 (2013a)

C. Qi, K.I. Öberg, D.J. Wilner, $\mathrm{H}_{2} \mathrm{CO}$ and $\mathrm{N}_{2} \mathrm{H}^{+}$in protoplanetary disks: Evidence for a CO-ice regulated chemistry. Astrophys. J. 765, 34 (2013b)

Y.L. Radeva, M.J. Mumma, B.P. Bonev, M.A. DiSanti, G.L. Villanuea, K. Magee-Sauer, E.L. Gibb, H.A. Weaver, The organic composition of comet C/2000 WM1 (LINEAR) revealed through infrared spectroscopy. Icarus 206, 764 (2010)

S.D. Rodgers, S.B. Charnley, Nitrogen isotopic fractionation of interstellar nitriles. Astrophys. J. 689, 1448 (2008) 
E. Roueff, J.C. Loison, K.M. Hickson, Isotopic fractionation of carbon, deuterium and nitrogen: a full chemical study. Astron. Astrophys. 576, 99 (2015)

K. Saigo, K. Tomisaka, T. Matsumoto, Evolution of first cores and formation of stellar cores in rotating molecular cloud cores. Astrophys. J. 674, 997 (2008)

C. Salyk, K.M. Pontoppidan, G.A. Blake, F. Lahuis, E.F. van Dishoeck, N.J. Evans II, $\mathrm{H}_{2} \mathrm{O}$ and $\mathrm{OH}$ gas in the terrestrial planet-forming zones of protoplanetary disks. Astrophys. J. 676, L49 (2008)

J. Schönke, W. Tscharnuter, Protostellar collapse of rotating cloud cores. Covering the complete first accretion period of the stellar core. Astron. Astrophys. 526, 139 (2011)

D. Semenov, D. Wiebe, Chemical evolution of turbulent protoplanetary disks and the solar nebula. Astrophys. J. Suppl. Ser. 196, 25 (2011)

D. Semenov, D. Wiebe, Th. Henning, Gas-phase CO in protoplanetary disks: A challenge for turbulent mixing. Astrophys. J. 647, L57 (2006)

Y. Shinnaka, H. Kawakita, H. Kobayashi, E. Jehin, J. Manfroid, D. Hutsemékers, C. Arpigny, Ortho-to-para abundance ratio (OPR) of ammonia in 15 comets: OPRs of ammonia versus ${ }^{14} \mathrm{~N} /{ }^{15} \mathrm{~N}$ ratios in $\mathrm{CN}$. Astrophys. J. 729, 81 (2011)

B. Silvi, A. Savin, Classification of chemical bonds based on topological analysis of electron localization functions. Nature 371, 683 (1994)

V. Taquet, P.S. Peters, C. Kahane, C. Ceccarelli, A. López-Sepulcre, C. Toubin, D. Duflot, L. Wiesenfeld, Water ice deuteration: A tracer of the chemical history of protostars. Astron. Astrophys. 550, 127 (2013)

V. Taquet, S.B. Charnley, O. Sipila, Multilayer formation and evaporation of deuterated ices in prestellar and protostellar cores. Astrophys. J. 791, 1 (2014)

P. Theulé, F. Duvernay, G. Danger, F. Borget, J.B. Bossa, B. Vinogradoff, F. Mispelaer, T. Chiavassa, Thermal reactions in interstellar ice: A step towards molecular complexity in the interstellar medium. Adv. Space Res. 52, 1567 (2013)

A.G.G.M. Tielens, Surface chemistry of deuterated molecules. Astron. Astrophys. 119, 177 (1983)

C. Tornow, P. Gast, I. Pelivan, S. Kupper, E. Kührt, U. Motschmann, Water formation in early solar nebula: I. Quasi-stationary cloud core. Planet. Space Sci. 98, 216 (2014a)

C. Tornow, P. Gast, U. Motschmann, S. Kupper, E. Kührt, I. Pelivan, Water formation in early solar nebula: II. Collapsing cloud core. Planet. Space Sci. 98, 233 (2014b)

N.J. Turner, K. Willacy, G. Bryden, H.W. Yorke, Turbulent mixing in the outer solar nebula. Astrophys. J. 639, 1218 (2006)

N.J. Turner, T. Sano, N. Dziourkevitch, Turbulent mixing and the dead zone in protostellar disks. Astrophys. J. 659, 729 (2007)

N.J. Turner, S. Fromang, C. Gammie, H. Klahr, G. Lesur, M. Wardle, X. Bai, Transport and accretion in planet-forming disks, in Protostars and Planets VI, ed. by H. Beuther, R. Klessen, C. Dullemond, Th. Henning (University of Arizona Press, Tucson, 2014), p. 411

A.I. Vasyunin, D.A. Wiebe, T. Birnstiel, S. Zhukovska, Th. Henning, C.P. Dullemond, Impact of grain evolution on the chemical structure of protoplanetary disks. Astrophys. J. 727, 76 (2011)

G.L. Villanueva, M.J. Mumma, B.P. Bonev, M.A. DiSanti, E.L. Gibb, H. Böhnhardt, M. Lippi, A sensitive search for deuterated water in Comet 8P/Tuttle. Astrophys. J. 690, 5 (2009)

G.J. Villanueva, M.J. Mumma, M.A. DiSanti, B.P. Bonev, E.L. Gibb, K. Magee-Sauer, G.A. Blake, C. Salyk, The molecular composition of comet C/2007 W1 (Boattini): Evidence of a peculiar outgassing and a rich chemistry. Icarus 216, 227 (2011)

R. Visser, E.F. van Dishoeck, J.H. Black, The photodissociation and chemistry of CO isotopologues: Applications to interstellar clouds and circumstellar disks. Astron. Astrophys. 503, 323 (2009a)

R. Visser, E.F. van Dishoeck, S.D. Doty, C.P. Dullemond, The chemical history of molecules in circumstellar disks. I. Ices. Astron. Astrophys. 495, 881-897 (2009b)

R. Visser, S.D. Doty, E.F. van Dishoeck, The chemical history of molecules in circumstellar disks. II. Gasphase species. Astron. Astrophys. 534, 132 (2011)

V. Wakelam, C. Vastel, Y. Aikawa, A. Coutens, S. Bottinelli, E. Caux, Chemical modeling of water deuteration in IRAS 16293-2422. Mon. Not. R. Astron. Soc. 445, 2854 (2012)

C. Walsh, T.J. Millar, H. Nomura, Molecular line emission from a protoplanetary disk irradiated externally by a nearby massive star. Astrophys. J. 766, L23 (2013)

C. Walsh, T.J. Millar, H. Nomura, E. Herbst, S. Widicus Weaver, Y. Aikawa, J.C. Laas, A.I. Vasyunin, Complex organic molecules in protoplanetary disks. Astron. Astrophys. 563, 33 (2014)

N. Watanabe, A. Kouchi, Ice surface reactions: A key to chemical evolution in space. Prog. Surf. Sci. 83, 439 (2008)

K. Willacy, The chemistry of multiply deuterated molecules in protoplanetary disks. I. The outer disk. Astrophys. J. 660, 44 (2007)

K. Willacy, W.D. Langer, The importance of photoprocessing in protostellar disks. Astrophys. J. 544, 903 (2000) 
K. Willacy, N.J. Turner, Turbulent mixing and the abundance of water ice at the snow-line of a protostellar disk (2015, in preparation)

K. Willacy, P.M. Woods, Deuterium chemistry in protoplanetary disks. II. The inner 30 AU. Astrophys. J. Lett. 703, 479 (2009)

K. Willacy, H.H. Klahr, T.J. Millar, Th. Henning, Gas and grain chemistry in a protoplanetary disk. Astron. Astrophys. 338, 995 (1998)

K. Willacy, W.D. Langer, M. Allen, G. Bryden, Turbulence-driven diffusion in protoplanetary disks: Chemical effects in the outer regions. Astrophys. J. 644, 1202 (2006)

E.S. Wirström, W.D. Geppert, A. Hjalmarson, C.M. Persson, J.H. Black, P. Bergman, T.J. Millar, M. Hamberg, E. Vigren, Observational tests of interstellar methanol formation. Astron. Astrophys. 533, 24 (2011)

E.S. Wirström, S.B. Charnley, M.A. Cordiner, S.N. Milam, Isotopic anomalies in primitive solar system matter: Spin-state-dependent fractionation of nitrogen and deuterium in interstellar clouds. Astrophys. J. 757, L11 (2012)

P.M. Woods, K. Willacy, Benzene formation in the inner regions of protostellar disks. Astrophys. J. 655 , L49 (2007)

P.M. Woods, K. Willacy, Carbon isotope fractionation in protoplanetary disks. Astrophys. J. 693, 1360 (2009)

C.E. Woodward, M.S. Kelley, D. Bockelée-Morvan, R.D. Gehrz, Water in comet C/2003 K4 (LINEAR) with Spitzer. Astrophys. J. 671, 1065 (2007)

S. Wyckoff, S.C. Tegler, L. Engel, Nitrogen abundance in comet Halley. Astrophys. J. 367, 641 (1991)

E.D. Young, Time-dependent oxygen isotopic effects of $\mathrm{CO}$ self-shielding across the solar protoplanetary disk. Earth Planet. Sci. Lett. 262, 468 (2007)

M. Yu, K. Willacy, S. Dodson-Robinson, N. Turner, N.J. Evans II, Peering into the giant planet forming region of an MRI-active disk with rare CO isotopologues. Astrophys. J. (2014, submitted)

H. Yurimoto, K. Kuramoto, Molecular cloud origin for the oxygen isotope heterogeneity in the solar system. Science 305, 1763 (2004)

H. Yurimoto, K. Kuramoto, A.N. Krot, E.R.D. Scott, J.N. Cuzzi, M.H. Thiemens, J.R. Lyons, Origin and evolution of oxygen isotopic compositions of the solar system, in Protostars and Planets V (University of Arizona Press, Tucson, 2007)

V. Zubko, E. Dwek, R.G. Arendt, Interstellar dust models consistent with extinction, emission, and abundance constraints. Astrophys. J. Suppl. Ser. 152, 211 (2004) 